\title{
An Expressive Mechanism for Auctions on the Web*
}

\author{
Paul Dütting ${ }^{\dagger} \quad$ Monika Henzinger ${ }^{\ddagger} \quad$ Ingmar Weber ${ }^{\S}$
}

November 15, 2011

\begin{abstract}
Auctions are widely used on the Web. Applications range from internet advertising to platforms such as eBay. In most of these applications the auctions in use are single/multiitem auctions with unit demand. The main drawback of standard mechanisms for this type of auctions, such as VCG and GSP, is the limited expressiveness that they offer to the bidders. The General Auction Mechanism (GAM) of [1] is taking a first step towards addressing the problem of limited expressiveness by computing a bidder optimal, envy free outcome for linear utility functions with identical slopes and a single discontinuity per bidder-item pair. We show that in many practical situations this does not suffice to adequately model the preferences of the bidders, and we overcome this problem by presenting the first mechanism for piece-wise linear utility functions with non-identical slopes and multiple discontinuities. Our mechanism runs in polynomial time. Like GAM it is incentive compatible for inputs that fulfill a certain nondegeneracy assumption, but our requirement is more general than the requirement of GAM. For discontinuous utility functions that are non-degenerate as well as for continuous utility functions the outcome of our mechanism is a competitive equilibrium. We also show how our mechanism can be used to compute approximately bidder optimal, envy free outcomes for a general class of continuous utility functions via piece-wise linear approximation. Finally, we prove hardness results for even more expressive settings.
\end{abstract}

\section{Introduction}

Auctions are widely used on the Web. They are, e.g., used by Google, Microsoft, and Yahoo! for search advertising [42. They are also used on platforms such as eBay for selling a broad variety of goods and services [43, 8]. In most of these applications the auctions in use are single/multi-item auctions with unit demand. The problem solved by these auctions is essentially a matching and pricing problem. In this problem $n$ bidders have to be matched to $k$ items. Each bidder has a utility function $u_{i, j}\left(p_{j}\right)$ that expresses his utility for being matched to item $j$ at price $p_{j}$. An outcome $(\mu, p)$ consisting of a matching $\mu$ and prices $p$ is said to be envy free if at the current prices every bidder (weakly) prefers the item that he is currently matched to over every other item 1 An envy free outcome $(\mu, p)$ is called bidder optimal if the utility of every bidder is at least as high as in every other envy free outcome $\left(\mu^{\prime}, p^{\prime}\right)$. Mechanisms that compute a bidder optimal, envy free outcome

\footnotetext{
*An extended abstract appeared in WWW'11.

†École Polytechnique Fédérale de Lausanne (EPFL), Station 14, CH-1015 Lausanne, Switzerland

Email: paul.duetting@epfl.ch.

${ }^{\ddagger}$ University of Vienna, Universitätsstraße 10/9, A-1090 Vienna, Austria

Email: monika.henzinger@univie.ac.at

§Yahoo! Research Barcelona, Avinguda Diagonal 177 8o, E-08018 Barcelona, Spain

Email: ingmar@yahoo-inc.com

${ }^{1}$ In the related literature (see, e.g., [1]) such outcomes are also referred to as stable.
} 
are desirable because they not only guarantee that everyone is "happy with what he gets", but also that everyone is "as happy as possible". From an economic point of view a bidder optimal, envy free outcome in which all unsold items have price zero is desirable because it represents a competitive equilibrium (or Walrasian equilibrium) [42].

\subsection{Limitations of Current Mechanisms}

Standard mechanisms for auctions on the web, such as First- and Second Price for single-item auctions and Vickrey Clarkes Groves (VCG) [49, 14, 33] and Generalized Second Price (GSP) [26, 47] for multi-item auctions, nicely fit into the above model. For linear utility functions with identical slopes, i.e., utilities of the form $u_{i, j}\left(p_{j}\right)=v_{i, j}-p_{j}$, where $v_{i, j}$ denotes bidder $i$ 's valuation for item $j$, the outcome of VCG, for example, is envy free and bidder optimal [38].

The main drawback of these standard mechanisms is the limited expressiveness that they offer to the bidders. In particular, (1) they typically limit what functions the bidders can use to express their utility for receiving a given item at a given price. In ad auctions, for example, some of the bidders may have per-click valuations, while others have per-impression valuations. Mechanisms that can handle either type exist (see, e.g., [26, 47] or [40, 28]), but mechanisms that can handle both types simultaneously are still rare [32]. Moreover, (2) they do not allow to express budgets. Budgets can either be soft (a limited amount of cash after which the bidder has to take out a loan) or hard (an upper bound on the price the bidder is willing to pay). Budgets are considered an important and difficult problem that recently has received a lot of attention (see, e.g., [11, 22]).

The General Auction Mechanism (GAM) of [1] is taking a first step towards addressing the problem of limited expressiveness by allowing the bidders to specify a maximum price for each item. That is, it finds a bidder optimal, envy free outcome for linear utilities with identical slopes and a single discontinuity per bidder-item pair. More specifically, the utility functions are of the form $u_{i, j}\left(p_{j}\right)=v_{i, j}-p_{j}$ if $p_{j} \leq m_{i, j}$ and $u_{i, j}\left(p_{j}\right)=-\infty$ otherwise, where $m_{i, j}$ is the bidder-item specific maximum price. GAM requires the input to be in general position (see [1] and Section 4 for a generalization of this concept). In general position it also preserves a desirable property of the original model. Namely, no bidder can misreport his valuations and/or maximum prices to achieve a higher utility. This makes it a (weakly) dominant strategy for the bidders to report truthfully. Mechanisms with this property are said to be incentive compatible (or strategy proof) [42].

Despite its generality GAM has three major limitations: (1) It can only handle linear utility functions with identical slopes. (2) It can only handle a single discontinuity with a jump to $-\infty$ per bidder-item pair. (3) It cannot handle non-linear utility functions. We illustrate why and when these shortcomings are problematic by means of three examples.

Example 1: Per-click vs. per-impression valuations (This example motivates linear utilities with non-identical slopes.) Consider an ad auction with bidders with per-click valuations $v_{i, j}^{\text {click }}$ and bidders with per-impression valuations $v_{i, j}^{i m p}$. The former are envy free if $u_{i}^{\text {click }} \geq v_{i, j}^{\text {click }}-p_{j}^{\text {click }}$ for all $j$ and the latter are envy free if $u_{i}^{i m p} \geq v_{i, j}^{i m p}-p_{j}^{i m p}$ for all $j$. Suppose that the mechanism collects per-click valuations and charges per-click prices. That is,

$$
u_{i, j}\left(p_{j}^{\text {click }}\right)=v_{i, j}^{\text {click }}-p_{j}^{\text {click }} .
$$

A bidder with per-impression valuations can translate his valuations into per-click valuations using the click trough rate $c t r_{i, j}$ as follows: $v_{i, j}^{c l i c k}=v_{i, j}^{i m p} / c t r_{i, j}$. That is, he reports $u_{i, j}\left(p_{j}^{c l i c k}\right)=$ $v_{i, j}^{i m p} / c t r_{i, j}-p_{j}^{c l i c k}$. Now suppose that given the per-click valuations, the mechanism computes an 


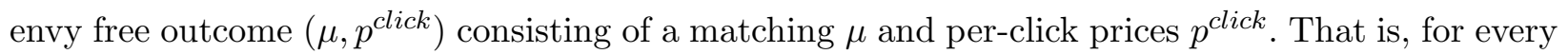
matched bidder-item pair $(i, j) \in \mu$ and all items $j^{\prime} \neq j$ we have:

$$
v_{i, j}^{\text {click }}-p_{j}^{\text {click }} \geq v_{i, j^{\prime}}^{\text {click }}-p_{j^{\prime}}^{\text {click }} .
$$

What we actually want for bidders $i$ with per-impression valuations $v_{i, j}^{i m p}$ is that for $(i, j) \in \mu$ and all $j^{\prime} \neq j$ :

$$
v_{i, j}^{i m p}-p_{j}^{i m p} \geq v_{i, j^{\prime}}^{i m p}-p_{j^{\prime}}^{i m p}
$$

But if we take (2), replace $v_{i, j}^{c l i c k}$ with $v_{i, j}^{i m p} / c t r_{i, j}, p_{j}^{c l i c k}$ with $p_{j}^{i m p} / c t r_{i, j}$, and multiply by $c t r_{i, j}$, then we get

$$
v_{i, j}^{i m p}-p_{j}^{i m p} \geq C \cdot\left(v_{i, j^{\prime}}^{i m p}-p_{j^{\prime}}^{i m p}\right),
$$

where $C=c t r_{i, j} / c t r_{i, j^{\prime}}$. That is, if $C<1$, then (41) is not strong enough to guarantee envy freeness for per-impression bidders. With non-identical slopes this can be sidestepped by having bidders with per-impression valuations report

$$
u_{i, j}\left(p_{j}^{c l i c k}\right)=v_{i, j}^{i m p}-c t r_{i, j} \cdot p_{j}^{c l i c k}
$$

In this case the outcome $\left(\mu, p^{\text {click }}\right)$ computed by the mechanism will be envy free for both types of bidders, i.e., the above problem does not arise.

Example 2: Soft and hard budgets (This example motivates piece-wise linear utilities with non-identical slopes and multiple discontinuities.) Suppose that bidder $i$ wants to buy a car on eBay. In the current system it would be dangerous for bidder $i$ to bid on more than one car at the same time due to the risk of winning and having to pay for several cars when one is already enough. With a GAM-like auction bidder $i$ could bid on many cars at the same time while still being guaranteed that he gets at most one.

With expensive items, such as cars, bidder $i$ 's valuation $v_{i, j}$ for item $j$ may exceed the amount $c$ of cash that he possesses. In this case $i$ might be willing to take out a loan if the price $p_{j}$ of item $j$ exceeds $c$. Assume that bidder $i$ is offered a loan with a maximum amount of $a$, an interest rate of $r$, and a fixed fee of $f$. Then bidder $i$ 's utility for item $j$ has the following form: (1) Because no interest is due for the first $c$ dollars the utility function drops linearly with a slope of -1 from 0 to $c$. (2) At $c$ it drops by the fixed fee $f$. (3) Afterwards, due to the interest rate $r$, every dollar spent causes $1+r$ dollars in actual cost. Hence the utility function drops linearly with a slope of $-(1+r)$ from $c$ to $c+a$.

In addition to the soft budget constraint $c$, bidder $i$ may have a hard budget constraint $b<c+a$, which is typically modeled by a jump to $-\infty$ at $p_{j}=b$. Hence bidder $i$ 's utility function for item $j$ ultimately looks like this: 


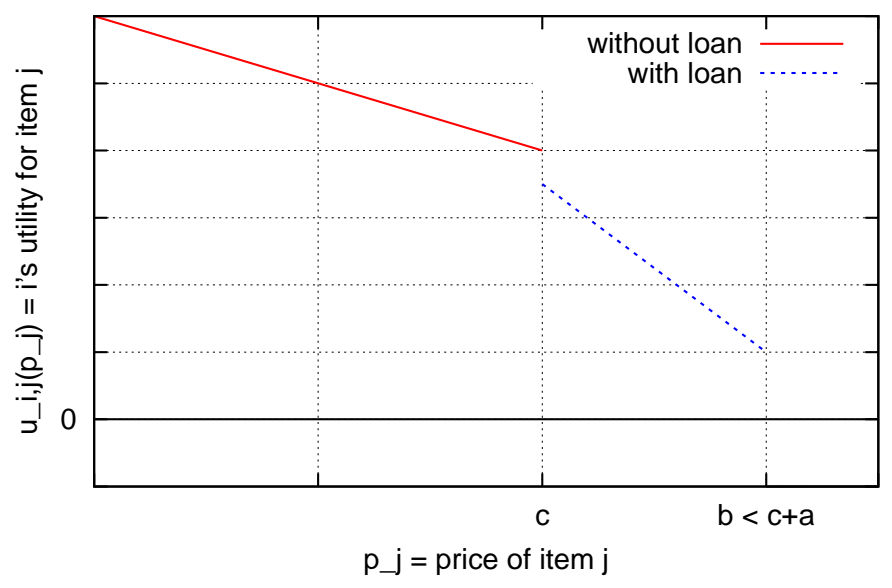

Figure 1: Bidder $i$ 's utility function for item $j$.

Note that import duties that apply when bidder $i$ wants to purchase car $j$ abroad and its price $p_{j}$ exceeds a certain threshold give rise to similar utility functions.

Example 3: Risk aversion (This example motivates arbitrary, non-linear utilities.) In the previous example bidder $i$ may also be faced with costs for uncertain events after the purchase, such as potential car repairs. A risk-averse buyer would have a utility function that drops superlinearly in the price as a higher price is associated with a higher risk of not having sufficient money to pay for the unforeseen events.

\subsection{Our Contributions}

We overcome the limitations of GAM as follows: (1) We present the first mechanism for piecewise linear utility functions with non-identical slopes and multiple discontinuities. The problem solved by our mechanism is more difficult than that solved by GAM as non-identical slopes require the prices to be increased by different amounts, and multiple discontinuities can cause previously matched bidders to become unmatched several times. Our mechanism is more expressive than GAM as it can simultaneously auction off items to bidders with per-click and per-impression valuations (Example 1), and it can handle a variety of soft and hard budget constraints (Example 2). The running time of our mechanism is polynomial in the number of bidders $n$ and items $k 2$ It is linear in the number of discontinuities $D$ and constant-slope intervals $T$ of the utility functions. (2) We precisely characterize under which conditions any mechanism that computes a bidder optimal outcome in this setting is incentive compatible. This characterization involves a generalization of the general position concept of [1]. For discontinuous utility functions that are non-degenerate as well as for continuous utility functions the outcome of our mechanism is a competitive equilibrium. (3) We show how to use our mechanism to compute a $\gamma$-bidder optimal, $\gamma$-envy free outcome for a general class of continuous utility functions via piece-wise linear approximation in time linear in $1 / \sqrt{\gamma}$. (4) Finally, we prove hardness results for two natural generalizations of our problem. In the first, the utilities may depend on the matching. In the second, they may depend on the whole vector of prices. In both cases computing a bidder optimal, envy free outcome is $\mathcal{N} \mathcal{P}$-hard.

\footnotetext{
${ }^{2}$ For $n>k$ it is linear in $n$.
} 


\subsection{Related Work}

Continuous utility functions For linear utility functions with identical slopes the underlying matching and pricing problem was first studied by Shapley and Shubik [45. They formulated the matching problem as a linear program and observed that the dual program yields envy free prices. With the help of this formulation they also proved the existence of an outcome with smallest prices/largest utilities, which is the bidder optimal outcome. Later Leonard [38] examined the incentives for misreporting the utility functions and found that the bidder optimal outcome is identical to the outcome of VCG [49, 14, 33] and therefore incentive compatible. The classic mechanism for linear utility functions with identical slopes is the so-called Multi-Item Auction of Demange et al. [19], which is a variant of the so-called Hungarian Method by Kuhn [37]. The basic idea of this mechanism is to start with prices all zero and to repeatedly raise the prices of overdemanded items by the same amount. This idea was generalized to continuous, piecewise linear utility functions with non-identical slopes by Alkan [3, 4], who showed that the prices of overdemanded items need to be raised by different amounts and that these amounts can be computed by solving a primal/dual problem. The existence of a bidder optimal outcome for more general, non-linear utility functions was shown by Demange and Gale [18] using a lattice-theoretic argument. They also proved that for continuous utility functions any mechanism that finds a bidder optimal outcome is incentive compatible. Recently, Alaei et al. [2] presented a novel, inductive characterization of the bidder optimal utilities/prices in this setting, which yields a constructive proof of existence. Although hardness results have been established for related problems (see, e.g., [21, 48]), it is not clear whether or under which conditions a bidder optimal outcome can be found efficiently for such general continuous utility functions.

Discontinuous utility functions The first to add a single discontinuity to otherwise linear utility functions with identical slopes were Aggarwal et al. [1]. They also gave a mechanism, which - for inputs in general position - is incentive compatible and finds a bidder optimal outcome in polynomial time. Similar results to that of [1] were obtained by [5, 6] and [25]. In [36] it was shown how to find the smallest envy free prices for a given matching. Recently, Chen et al. [12] gave a polynomial-time mechanism for consistent utility functions. Note that all these results either assume identical slopes [1, 6, 25, 36], just a single discontinuity [1, 6, 25, 36, 12, or both. Also note that the piece-wise linear utility functions with non-identical slopes and multiple discontinuities that we study here are not consistent. The existence of a bidder optimal, envy free outcome for more general, non-linear utility functions with multiple discontinuities was established in [24], but just as in the continuous case no polynomial-time mechanism is known for such general discontinuous utility functions.

Externalities Our hardness results rely on the fact that bidder $i$ 's utility for being matched to item $j$ may depend on (a) who is assigned which item or (b) the prices of the other items. Such dependencies are referred to as externalities. The "classic" result here is due to Jehiel et al. [35], who presented a revenue maximizing auction for the sale of a single item when bidders who do not acquire the item for sale incur a bidder-dependent externality. Other related results are [7] and [30]. The former analyzes Nash equilibria of so-called social context games in which utilities are computed based on an undirected neighborhood graph among players and aggregation functions. The latter proves that the following winner determination problem, which, e.g, arises in the pay-per lead model, is $\mathcal{N} \mathcal{P}$-hard and hard to approximate: Choose a set $S$ of at most $k$ advertisers from a set of $n$ advertisers, each with a private value $v_{i}$ and a random quality variable $q_{i}$ to maximize the expected value $v(S)=\sum_{i \in S} v_{i} \cdot \operatorname{Pr}\left[\forall j \in S \cup\{0\}: q_{i} \geq q_{j}\right]$, where $q_{0}$ is the quality of the best outside 
option. The externality is thus encoded in the choice of $S$ and the fact that the distributions from which the $q_{i}$ are drawn need not be independent. Our problem is different as there is not a single winner and the externality is either encoded in the matching or the prices.

\subsection{Overview of Techniques}

Our main result, the generalization of GAM, draws from the rich literature on matching and pricing problems in various ways: First, it uses the same terminology (e.g., strict overdemand, alternating path, alternating tree) and exploits the same link to Hall's theorem [34] that was already used in [19] and in [37]. It also borrows from [3, 4], in which the computation of price increases for continuous, piece-wise linear utility functions with non-identical slopes is formulated as a primal/dual problem, although we refine this approach to significantly improve upon the running time and extend it to discontinuous utility functions 3 Finally, our analysis follows a similar approach as [1] to cope with the fact that in the presence of discontinuities bidder optimality and incentive compatibility no longer coincide by characterizing inputs for which this is still the case.

On a conceptual level our main achievement is a decoupling of the argument for bidder optimality from the argument for incentive compatibility. On a technical level it is that we can show bidder optimality even if discontinuities require previously matched bidders to be unmatched. We achieve this through a refinement of Alkan's technique for increasing the prices when slopes are non-identical (Lemma 7) with a novel argument that shows that all price increases by the mechanism are necessary, even if discontinuities are reached and bidders become unmatched (Lemma 8). Together these lemmata allow us to prove bidder optimality even for inputs which make it necessary to unmatch previously matched bidders. Note that neither [3, 4] nor [1] have to unmatch bidders, because they either assume continuity or restrict themselves to inputs in general position.

Our new insight for incentive compatibility is that if for each price increase at most one utility function reaches a discontinuity, then bidders never get unmatched (Lemma 9). We also observe that in this case (a) all items with price $p_{j}>r_{j}$, where $r_{j}$ denotes an item-dependent reserve price, are matched, and (b) at least one item $j$ that is matched has $p_{j}=r_{j}$ (Lemma 9). We then use a variant of Hwang's Lemma (Lemma 10) to prove that whenever (a) and (b) are satisfied any mechanism that computes a bidder optimal outcome is incentive compatible. Finally, we formulate a condition on the input that guarantees that for each price increase at most one discontinuity is reached. Following Aggarwal et al. [1] we say that inputs that satisfy this condition are in general position, but our condition on the input is more general than that of [1] as it applies to piece-wise linear utility functions with non-identical slopes and multiple discontinuities.

Concerning general continuous utility functions it is intuitively obvious that the solution to a close enough piece-wise linear approximation cannot be far worse than the solution to the original problem. The difficulty here is to get a running time that is polynomial in $1 / \gamma$, where $\gamma$ is the maximal distance between the resulting bidder optimal utilities for the approximate utility functions and for the original utility functions. In fact, it is not difficult to show that $O(1 / \sqrt{\epsilon})$ linear segments suffice to ensure that the maximum distance between the approximate utility functions and the original utility functions is at most $\epsilon$ (Lemma 11). The hard part is to show that the distance $\gamma$ between the resulting bidder optimal utilities scales linearly in $\epsilon$ (Lemma 12).

We establish the hardness result for utility functions that depend on the matching through a reduction from 3-SAT [16] and the hardness result for utility functions that depend on the whole vector of prices through a reduction from MAX-2-NASH [31, 15], 4h The reduction from MAX-2NASH represents a novel and interesting link between the matching and pricing problem studied

\footnotetext{
${ }^{3}$ In [3, 4] the running time is stated as $O\left(n^{2} \cdot k^{4} \cdot \prod_{i, j} t_{i, j}\right)$, where $t_{i, j}$ is the number of linear segments of $u_{i, j}(\cdot)$.

${ }^{4}$ MAX-2-NASH: Find a Nash equilibrium of a 2-player game which maximizes the sum of the utilities.
} 
here and the problem of computing Nash equilibria, whose computational complexity has been settled only recently [17, 13].

\section{Problem Statement}

We are given a set $I$ of $n$ bidders and a set $J$ of $k$ items. The set of items $J$ contains a dedicated dummy item that we denote $j_{0}$. For each bidder $i$ we are given a constant $o_{i}$, called the outside option, which is the utility that bidder $i$ derives from not getting any non-dummy item. For each item $j$ we are given a constant $r_{j} \geq 0$, called the reserve price, which is a lower bound on $p_{j}$. Finally, for each bidder-item pair $(i, j)$ we are given a utility function $u_{i, j}\left(p_{j}\right)$, where $p_{j}$ denotes the price of item $j$. The utility functions are piece-wise linear. That is, each $u_{i, j}(\cdot)$ is composed of $t_{i, j}$ linear segments

$$
u_{i, j}^{(t)}\left(p_{j}\right)=v_{i, j}^{(t)}-c_{i, j}^{(t)} \cdot p_{j} \text { for } p_{j} \in\left[s_{i, j}^{(t)}, e_{i, j}^{(t)}\right),
$$

where $t \in\left\{1, \ldots, t_{i, j}\right\}, s_{i, j}^{(1)}=r_{j}, e_{i, j}^{\left(t_{i, j}\right)}=\infty, s_{i, j}^{(t)}<e_{i, j}^{(t)}(\forall t)$, and $e_{i, j}^{(t)}=s_{i, j}^{(t+1)}\left(\forall t \neq t_{i, j}\right)$. Where possible we omit $(t)$ to improve readability. We make the following assumptions concerning the utility functions: (1) They are strictly monotonically decreasing. (2) They need not be globally continuous. (3) For every bidder-item pair $(i, j)$ there exists a threshold value $\bar{p}_{i, j}$ such that $u_{i, j}\left(\bar{p}_{i, j}\right) \leq o_{i}$. (4) The utility functions $u_{i, j_{0}}(\cdot)$ for the dummy item $j_{0}$ are of the form $u_{i, j_{0}}\left(p_{j_{0}}\right)=o_{i}-p_{j_{0}}$ for $p_{j_{0}} \in[0, \infty)$ and $r_{j_{0}}=0.5$

We want to compute (1) a subset $\mu \subseteq I \times J$ of the bidder-item pairs, in which (a) every bidder $i$ appears in exactly one pair $(i, j) \in \mu$ and (b) every non-dummy item $j \neq j_{0}$ appears in at most one pair. We refer to this set as matching, even though multiple bidders $i$ can be matched to the dummy item $j_{0}$. We also want to compute (2) per-item prices $p=\left(p_{1}, . ., p_{k}\right)$. We refer to the pair $(\mu, p)$ as outcome. An outcome $(\mu, p)$ is feasible if

$$
p_{j_{0}}=0 \text { and } p_{j} \geq r_{j} \text { for all } j \neq j_{0} \text {. }
$$

We say that prices with this property are feasible. In the remainder of this paper whenever we refer to prices we mean feasible prices. An outcome is envy free if it is feasible and for all $i$ and $(i, j) \in I \times J$,

$$
u_{i, \mu(i)}\left(p_{\mu(i)}\right) \geq u_{i, j}\left(p_{j}\right)
$$

where $\mu(i)$ denotes the item bidder $i$ is matched to. An outcome $(\mu, p)$ is bidder optimal if it is envy free and for every bidder $i$ and every envy free outcome $\left(\mu^{\prime}, p^{\prime}\right)$ we have

$$
u_{i, \mu(i)}\left(p_{\mu(i)}\right) \geq u_{i, \mu^{\prime}(i)}\left(p_{\mu^{\prime}(i)}^{\prime}\right) .
$$

Our goal is to compute a bidder optimal outcome.

\section{Mechanism}

In this section we describe and analyze our mechanism for piece-wise linear utilities. We proceed as follows: First, we show how to reduce the problem of finding a bidder optimal outcome for an input with reserve prices to the problem of finding such an outcome for a different input in which the

\footnotetext{
${ }^{5}$ Note that this definition guarantees that in every envy free outcome every bidder $i$ has utility at least $o_{i}$.
} 
reserve prices are all zero. Second, we prove that the bidder optimal outcome has minimal prices among all envy free outcomes. We then formulate the problem as a graph problem. This allows us to define strict overdemand and to prove that an envy free outcome exists if and only if no set of items is strictly overdemanded using Hall's Theorem [34]. Our mechanism, which can be seen as an algorithmic version of Hall's Theorem [34], starts with prices all zero and iteratively raises the prices of strictly overdemanded items. To ensure minimality of the resulting prices it does this in an envy free and overdemand preserving manner.

Standard form We say that the input is in standard form if $r_{j}=0$ for all $j$. The following lemma shows that we can w.l.o.g. assume that the input is in standard form as for any problem instance that is not in standard form there is a linear-time reduction to an instance in standard form. This reduction is similar to the reduction described in 3. The lemma also shows that a sufficient condition for an outcome $\left(\mu^{*}, p^{*}\right)$ to be bidder optimal is that the prices $p^{*}$ are the minimum prices at which an envy free outcome exists. This was already known for continuous utility functions (see, e.g., [18]), but it is a novel observation for discontinuous utility functions. Moreover, unlike in the continuous case, the reverse need not be true for discontinuous utility functions.

Lemma 1. We have:

1. If the outcome $(\mu, p)$ is bidder optimal for $u_{i, j}^{\prime}\left(p_{j}\right)=u_{i, j}\left(p_{j}+r_{j}\right)$ and $r_{j}^{\prime}=0$, then the outcome $\left(\mu, p^{\prime}\right)$ with $p_{j}^{\prime}=p_{j}+r_{j}$ is bidder optimal for $u_{i, j}\left(p_{j}\right)$ and $r_{j}$.

2. If the outcome $\left(\mu^{*}, p^{*}\right)$ is envy free and $p_{j}^{*} \leq p_{j}$ for all items $j$ and every envy free outcome $(\mu, p)$, then $\left(\mu^{*}, p^{*}\right)$ is bidder optimal.

Proof. To 1.: The outcome $\left(\mu, p^{\prime}\right)$ is feasible for $u_{i, j}\left(p_{j}\right)$ and $r_{j}$ because $p_{j_{0}}^{\prime}=p_{j_{0}}+r_{j_{0}}=p_{j_{0}}=0$ and $p_{j}^{\prime}=p_{j}+r_{j} \geq r_{j}$ for all $j \neq j_{0}$. It is envy free because $u_{i, \mu(i)}\left(p_{\mu(i)}^{\prime}\right)=u_{i, \mu(i)}\left(p_{\mu(i)}+r_{\mu(i)}\right)=$ $u_{i, \mu(i)}^{\prime}\left(p_{\mu(i)}\right) \geq u_{i, j}^{\prime}\left(p_{j}\right)=u_{i, j}\left(p_{j}+r_{j}\right)=u_{i, j}\left(p_{j}^{\prime}\right)$ for all $i \in I$ and all $j \in J$. To see that it is also bidder optimal, suppose for a contradiction that it is not, i.e., there exists an outcome $\left(\mu^{\prime \prime}, p^{\prime \prime}\right)$ that is envy free for $u_{i, j}\left(p_{j}\right)$ and $r_{j}$ and has $u_{i, \mu^{\prime \prime}(i)}\left(p_{\mu^{\prime \prime}(i)}^{\prime \prime}\right) \geq u_{i, \mu(i)}\left(p_{\mu(i)}^{\prime}\right)$ for all $i \in I$ and all $j \in J$; with at least one inequality strict. But then the outcome $\left(\mu^{\prime \prime}, p^{\prime \prime \prime}\right)$ with $p_{j}^{\prime \prime \prime}=p_{j}^{\prime \prime}-r_{j}$ for all $j$ is (a) feasible for $u_{i, j}^{\prime}\left(p_{j}\right)$ and $r_{j}^{\prime}=0$ because $p_{j_{0}}^{\prime \prime \prime}=p_{j_{0}}^{\prime \prime}-r_{j_{0}}=0$ and $p_{j}^{\prime \prime \prime}=p_{j}^{\prime \prime}-r_{j} \geq 0$ and (b) envy free for $u_{i, j}^{\prime}\left(p_{j}\right)$ and $r_{j}^{\prime}=0$ because $u_{i, \mu^{\prime \prime \prime}(i)}^{\prime}\left(p_{\mu^{\prime \prime}(i)}^{\prime \prime \prime}\right)=u_{i, \mu^{\prime \prime}(i)}^{\prime}\left(p_{\mu^{\prime \prime}(i)}^{\prime \prime}-r_{\mu^{\prime \prime}(i)}\right)=u_{i, \mu^{\prime \prime}(i)}\left(p_{\mu^{\prime \prime}(i)}^{\prime \prime}\right) \geq$ $u_{i, j}\left(p_{j}^{\prime \prime}\right)=u_{i, j}^{\prime}\left(p_{j}^{\prime \prime}-r_{j}\right)=u_{i, j}^{\prime}\left(p_{j}^{\prime \prime \prime}\right)$ for all $i \in I$ and all $j \in J$. Hence $u_{i, \mu^{\prime \prime}(i)}^{\prime}\left(p_{\mu^{\prime \prime}(i)}^{\prime \prime \prime}\right)=u_{i, \mu^{\prime \prime}(i)}\left(p_{\mu^{\prime \prime}(i)}^{\prime \prime}\right) \geq$ $u_{i, \mu(i)}\left(p_{\mu(i)}^{\prime}\right)=u_{i, \mu(i)}^{\prime}\left(p_{\mu(i)}\right)$ for all $i \in I$ and all $j \in J$; with at least one of the inequalities strict. This contradicts the bidder optimality of $(\mu, p)$ for $u_{i, j}^{\prime}(\cdot)$ and $r_{j}^{\prime}=0$.

To 2.: By contradiction assume that there is an envy free outcome $\left(\mu^{\prime}, p^{\prime}\right)$ with $u_{i, \mu^{\prime}(i)}\left(p_{\mu^{\prime}(i)}^{\prime}\right)>$ $u_{i, \mu^{*}(i)}\left(p_{\mu^{*}(i)}^{*}\right)$ for some bidder $i$. Since $\left(\mu^{*}, p^{*}\right)$ is envy free, we have $u_{i, \mu^{*}(i)}\left(p_{\mu^{*}(i)}^{*}\right) \geq u_{i, \mu^{\prime}(i)}\left(p_{\mu^{\prime}(i)}^{*}\right)$. It follows that $u_{i, \mu^{\prime}(i)}\left(p_{\mu^{\prime}(i)}^{\prime}\right)>u_{i, \mu^{\prime}(i)}\left(p_{\mu^{\prime}(i)}^{*}\right)$, which implies $p_{\mu^{\prime}(i)}^{\prime}<p_{\mu^{\prime}(i)}^{*}$. Contradiction!

Graph-theoretic formulation Next we formulate the problem of computing an envy free outcome as a graph problem. Central to this formulation is the first choice graph $G_{p}=\left(I \cup J, F_{p}\right)$ at prices $p$, which consists of one node per bidder $i$, one node per item $j$, and an edge from $i$ to $j$ if and only if item $j$ gives bidder $i$ the highest utility at the current prices. That is, $u_{i, j}\left(p_{j}\right) \geq u_{i, k}\left(p_{k}\right)$ for all $k$. For $i \in I$ we define $F_{p}(i)=\left\{j: \exists(i, j) \in F_{p}\right\}$ and similarly $F_{p}(j)=\left\{i: \exists(i, j) \in F_{p}\right\}$. Analogously, for $T \subseteq I$ we define $F_{p}(T)=\cup_{i \in T} F_{p}(i)$ and for $S \subseteq J$ we define $F_{p}(S)=\cup_{j \in S} F_{p}(j)$. Based on the first choice graph we define strict overdemand: A set of non-dummy items $S \subseteq J \backslash\left\{j_{0}\right\}$ is strictly overdemanded at prices $p$ with respect to the set of bidders $T \subseteq I$ if (a) $F_{p}(T) \subseteq S$ and (b) for all $R \subseteq S$ with $R \neq \emptyset:\left|F_{p}(R) \cap T\right|>|R|$. A set of non-dummy items $S \subseteq J \backslash\left\{j_{0}\right\}$ is 
strictly overdemanded, if it is strictly overdemanded with respect to some set of bidders $T \subseteq I$. Our definition of strict overdemand is stronger than the definition of overdemand [19], which only requires that the number of bidders $T$ demanding only items in the set $S$ is greater than the number of items in the set. It is different from the notion of minimal overdemand [19, which requires that no subset is overdemanded. It is also different from the notion of directional overdemand in [4]. The advantage of our definition will become clear in the next subsection. The following lemma is established using Hall's Theorem [34] and the fact that a strictly overdemanded set of items exists if and only if an overdemanded set of items exists.

Lemma 2. The following statements are equivalent:

1. The outcome $(\mu, p)$ is envy free.

2. There exists a matching $\mu$ in $G_{p}$.

3. No set of items $S \subseteq J \backslash\left\{j_{0}\right\}$ is strictly overdemanded at prices $p$.

Proof. 1. $\Leftrightarrow 2 .:$ An outcome $(\mu, p)$ is envy free if and only if (a) $p_{j_{0}}=0$ and $p_{j} \geq 0$ for $j \neq j_{0}$ and (b) $u_{i, \mu(i)}\left(p_{\mu(i)}\right) \geq u_{i, j}\left(p_{j}\right)$ for all $(i, j) \in I \times J$ if and only if all edges $(i, j) \in \mu$ belong to the first choice graph $G_{p}$ at prices $p$ with $p_{j_{0}}=0$ and $p_{j} \geq 0$ for $j \neq j_{0}$.

2. $\Leftrightarrow 3 .:$ By Hall's Theorem [34] there exists a matching $\mu$ in the first choice graph at prices $p$ with $p_{j_{0}}=0$ and $p_{j} \geq 0$ for $j \neq j_{0}$ if and only if $\forall T \subseteq I:\left|F_{p}(T)\right| \geq|T|$ or $j_{0} \in F_{p}(T)$.

2. $\Rightarrow 3 .:$ Assume that $\forall T \subseteq I:\left|F_{p}(T)\right| \geq|T|$ or $j_{0} \in F_{p}(T)$. For a contradiction suppose that there exists a set of items $S^{\prime} \subseteq J \backslash\left\{j_{0}\right\}$ that is strictly overdemanded with respect to the set of bidders $T^{\prime}$, i.e., (a) $F_{p}\left(T^{\prime}\right) \subseteq S^{\prime}$ and (b) $\forall R \subseteq S^{\prime}:\left|F_{p}(R) \cap T^{\prime}\right|>|R|$. Let $T^{\prime \prime}=F_{p}\left(S^{\prime}\right) \cap T^{\prime}$. From (a) we know that $\left|F_{p}\left(T^{\prime}\right)\right| \leq\left|S^{\prime}\right|$. From (b) we know that $\left|T^{\prime \prime}\right|=\left|F_{p}\left(S^{\prime}\right) \cap T^{\prime}\right|>\left|S^{\prime}\right|$. Since $T^{\prime \prime}=F_{p}\left(S^{\prime}\right) \cap T^{\prime} \subseteq T^{\prime}$, we have $F_{p}\left(T^{\prime \prime}\right) \subseteq F_{p}\left(T^{\prime}\right)$ and, thus, $\left|F_{p}\left(T^{\prime \prime}\right)\right| \leq\left|F_{p}\left(T^{\prime}\right)\right|$. It follows that $\left|T^{\prime \prime}\right|>\left|F_{p}\left(T^{\prime \prime}\right)\right|$. Since $F_{p}\left(T^{\prime \prime}\right) \subseteq F_{p}\left(T^{\prime}\right) \subseteq S^{\prime} \subseteq J \backslash\left\{j_{0}\right\}$, we have $j_{0} \notin F_{p}\left(T^{\prime \prime}\right)$. Hence for $T^{\prime \prime}$ neither $\left|F_{p}\left(T^{\prime \prime}\right)\right| \geq\left|T^{\prime \prime}\right|$ nor $j_{0} \in F_{p}\left(T^{\prime \prime}\right)$. Contradiction!

$2 . \Leftarrow 3 .:$ Assume that no set of items $S^{\prime} \subseteq J \backslash\left\{j_{0}\right\}$ is strictly overdemanded. For a contradiction suppose that there exists $T^{\prime \prime} \subseteq I:\left|F_{p}\left(T^{\prime \prime}\right)\right|<\left|T^{\prime \prime}\right|$ and $j_{0} \notin F_{p}\left(T^{\prime \prime}\right)$. Consider the smallest such $T^{\prime \prime}$ and some $i \in T^{\prime \prime}$. For all proper subsets $T^{\prime \prime \prime} \subset T^{\prime \prime}:\left|F_{p}\left(T^{\prime \prime \prime}\right)\right| \geq\left|T^{\prime \prime \prime}\right|$. Hence all bidders in $T^{\prime \prime} \backslash\{i\}$ can be matched to items in $F_{p}\left(T^{\prime \prime} \backslash\{i\}\right)$ by Hall's Theorem [34. Let $\mu^{\prime}$ be such a matching. Let $\mu^{\prime}\left(T^{\prime \prime} \backslash\{i\}\right)$ denote the items matched to bidders in $T^{\prime \prime} \backslash\{i\}$ under $\mu^{\prime}$. Compute a maximal alternating tree $\mathcal{T}$ with respect to $\mu^{\prime}$ with root $i$. Denote the bidders and items in this tree by $T^{\prime} \subseteq T^{\prime \prime}$ and $S^{\prime}=F_{p}\left(T^{\prime}\right) \subseteq F_{p}\left(T^{\prime \prime}\right)$. It follows that (a) $j_{0} \notin S^{\prime}$ because $S^{\prime}=F_{p}\left(T^{\prime}\right) \subseteq$ $F_{p}\left(T^{\prime \prime}\right) \subseteq J \backslash\left\{j_{0}\right\}$ and (b) all items in $F_{p}\left(T^{\prime \prime}\right) \supseteq F_{p}\left(T^{\prime}\right)=S^{\prime}$ are matched because otherwise $\left|F_{p}\left(T^{\prime \prime}\right)\right|>\left|\mu^{\prime}\left(T^{\prime \prime}\right)\right|=\left|\mu^{\prime}\left(T^{\prime \prime} \backslash\{i\}\right)\right|=\left|T^{\prime \prime} \backslash\{i\}\right|=\left|T^{\prime \prime}\right|-1$, i.e., $\left|F_{p}\left(T^{\prime \prime}\right)\right| \geq\left|T^{\prime \prime}\right|$. Hence Lemma 3 shows that $S^{\prime}$ is strictly overdemanded with respect to $T^{\prime}$. Contradiction!

Alternating paths and trees To identify strictly overdemanded items our mechanism makes use of alternating paths and trees: Let $\mu$ be a partial matching. That is, a matching in which not all of the bidders have to be matched. An alternating path $\mathcal{P}$ with respect to $\mu$ in the first choice graph $G_{p}$ at prices $p$ from an unmatched bidder $i_{0}$ to some item or bidder $j$ is a sequence of edges that alternates between unmatched and matched edges and in which all items except $j$ are non-dummy items. An alternating tree $\mathcal{T}$ with respect to $\mu$ with root $i_{0}$ is a tree in the first choice graph $G_{p}$ at prices $p$ which is rooted at an unmatched bidder $i_{0}$ and in which all paths from the root $i_{0}$ to a leaf $j$ are alternating. An alternating tree is maximal if the first choice items of all bidders in the tree are contained in the tree and all matched items in the tree are matched to bidders in the tree. Formally: If $T \subseteq I$ and $S \subseteq J$ are the bidders and items in the tree $\mathcal{T}$, then $F_{p}(T) \subseteq S$ and $\mu(S) \subseteq T$. The fact that a partial matching can be augmented along an alternating path from an 
unmatched bidder to an unmatched item has been used before (see, e.g., [19]). The new insight of the following lemma is that there is a close correspondence between maximal alternating trees and our definition of strict overdemand.

Lemma 3. For any maximal alternating tree $\mathcal{T}$ with respect to $\mu$ with root $i_{0}$ in $G_{p}$, we have:

1. If the dummy item $j_{0}$ or some unmatched item $j \neq j_{0}$ is contained in $\mathcal{T}$, then the matching $\mu$ can be augmented along an alternating path $\mathcal{P}$ from $i_{0}$ to $j_{0}$ resp. $j$.

2. If all items $S$ in $\mathcal{T}$ are non-dummy items and matched, then $S$ is strictly overdemanded with respect to the bidders $T$ in the tree and $|T|=|S|+1$.

Proof. To 1.: The path $P$ is the path in the maximal alternating tree $\mathcal{T}$ that leads from $i_{0}$ to $j_{0}$ resp. $j$. All bidders on this path except $i_{0}$ are incident to two edges, one matched and one unmatched, and they are indifferent between the two. Hence we can swap the matched and unmatched edges along $P$ to augment the size of the matching by one.

To 2.: We know that $j_{0} \notin S$. From the maximality of the tree $\mathcal{T}$ we get $F_{p}(T) \subseteq S$. We still have to show that for all $R \subseteq S:\left|F_{p}(R) \cap T\right|>|R|$. For every item set $R \subseteq S$ we know that there exists a node $x \in R$ such that no other node of $R$ lies on the path $P$ from $x$ to the root $i_{0}$. Note that $x$ is not the root because the root does not belong to $R$. Let $y$ be the neighbor of $x$ on $P$. Then $y$ belongs to $F_{p}(R) \cap T$, but it is not matched to any node in $R$. Thus, counting the nodes matched to nodes in $R$ and $y$, there are at least $|R|+1$ nodes in $F_{p}(R) \cap T$.

Envy free price increase Once we have identified a strictly overdemanded set of items we need to determine how to increase the prices in the set: A price increase $d$ is a $k$-dimensional vector with entries $d_{j}$ for $j \in\{1, \ldots, k\}$. A price increase is envy free with respect to a set of first choice edges $E \subseteq F_{p} \backslash\left(I \times\left\{j_{0}\right\}\right)$ at prices $p$ if (a) $d_{j}>0$ for all $j$ such that there is a bidder $i$ with $(i, j) \in E$ and $d_{j}=0$ otherwise, and (b) $u_{i, j}\left(p_{j}+\lambda \cdot d_{j}\right) \geq u_{i, k}\left(p_{k}+\lambda \cdot d_{k}\right)$ for all $(i, j) \in E$, all $(i, k) \in F_{p}$, and all sufficiently small $\lambda>0$. Note that it is sufficient to require (b) for all $(i, k) \in F_{p}$ and not all $(i, k) \in I \times J$, because for every $(i, k) \in(I \times J) \backslash F_{p}$ we have $u_{i, j}\left(p_{j}\right)>u_{i, k}\left(p_{k}\right)$ and, thus, $u_{i, j}\left(p_{j}+\lambda \cdot d_{j}\right) \geq u_{i, k}\left(p_{k}+\lambda \cdot d_{k}\right)$ holds already. Also note that because for no bidder $i$ we have $\left(i, j_{0}\right) \in E$ any envy free price increase has $d_{j_{0}}=0$. Our definition of envy free price increase is similar to the definition of a competitive direction in [3]. The next two lemmata are proved in [3] for competitive directions and continuous utility functions, we generalize them to envy free price increases and discontinuous utility functions. The first lemma is an immediate consequence of the definition of envy free price increases. The second lemma gives a sufficient and necessary condition for a price increase $d \neq 0$ to be envy free for a set of first choice edges $E \subseteq F_{p}$. It shows that a price increase $d$ is envy free for a first choice edge $(i, j) \in F_{p}$ if and only if the "utility drop" $c_{i, j} \cdot d_{j}$ on this edge is minimal across the first choice edges $(i, k) \in F_{p}$ incident to $i$. We exploit this characterization in the computation of price increases described in the next subsection.

Lemma 4. If $d$ is an envy free price increase with respect to the set of first choice edges $E \subseteq$ $F_{p} \backslash\left(I \times\left\{j_{0}\right\}\right)$ at prices $p$, then $E$ belongs to the set of first choice edges at prices $p+\lambda \cdot d$ for all sufficiently small $\lambda>0$.

Proof. Consider an arbitrary bidder-item pair $(i, j) \in E$. If $(i, k) \in F_{p}$ : Since $d$ is envy free for $E$ and $(i, j) \in E$, we have $u_{i, j}\left(p_{j}+\lambda \cdot d_{j}\right) \geq u_{i, k}\left(p_{k}+\lambda \cdot p_{k}\right)$ for all $\lambda>0$ sufficiently small. If $(i, k) \in(I \times J) \backslash F_{p}$ : Since $u_{i, j}\left(p_{j}\right)>u_{i, k}\left(p_{k}\right)$, we have $u_{i, j}\left(p_{j}+\lambda \cdot d_{j}\right) \geq u_{i, k}\left(p_{k}+\lambda \cdot d_{k}\right)$ for all $\lambda>0$ sufficiently small 6 We conclude that $u_{i, j}\left(p_{j}+\lambda \cdot d_{j}\right) \geq u_{i, k}\left(p_{k}+\lambda \cdot d_{k}\right)$ for all $(i, k) \in I \times J$

\footnotetext{
${ }^{6}$ There is no discontinuity in the utility function $u_{i, j}(\cdot)$ within the range $\left[p_{j}, p_{j}+\lambda \cdot d_{j}\right]$ for all $\lambda>0$ sufficiently small because the utility function $u_{i, j}(\cdot)$ is locally right-continuous.
} 
for all $\lambda>0$ sufficiently small. Since $d_{j} \geq 0$ for all $j$ s.t. there is a bidder $i$ with $(i, j) \in E$ and $d_{j}=0$ otherwise, we have $p_{j_{0}}+\lambda \cdot d_{j_{0}}=p_{j_{0}}=0$ and $p_{j}+\lambda \cdot p_{j} \geq 0$ for all $j \neq j_{0}$.

Lemma 5. A price increase $d \neq 0$ is envy free for the set of first choice edges $E \subseteq F_{p}$ at prices $p$ if and only if $c_{i, j} \cdot d_{j} \leq c_{i, k} \cdot d_{k}$ for all $(i, j) \in E \subseteq F_{p}$ and all $(i, k) \in F_{p}$.

Proof. For the if-part assume that $u_{i, j}\left(p_{j}+\lambda \cdot d_{j}\right) \geq u_{i, k}\left(p_{k}+\lambda \cdot d_{k}\right)$ for all sufficiently small $\lambda>0$, all $(i, j) \in S \subseteq F_{p}$, and all $(i, k) \in F_{p}$. Consider arbitrary edges $(i, j) \in S \subseteq F_{p}$ and $(i, k) \in F_{p}$. By piece-wise linearity, $u_{i, j}\left(p_{j}+\lambda \cdot d_{j}\right)=u_{i, j}\left(p_{j}\right)-c_{i, j} \cdot \lambda \cdot d_{j}$ and $u_{i, k}\left(p_{k}+\lambda \cdot d_{k}\right)=u_{i, k}\left(p_{k}\right)-c_{i, k} \cdot \lambda \cdot d_{k}$ for all sufficiently small $\lambda>0$. From this and the fact that $u_{i, j}\left(p_{j}+\lambda \cdot d_{j}\right) \geq u_{i, k}\left(p_{k}+\lambda \cdot d_{k}\right)$ it follows that $u_{i, j}\left(p_{j}\right)-c_{i, j} \cdot \lambda \cdot d_{j} \geq u_{i, k}\left(p_{k}\right)-c_{i, k} \cdot \lambda \cdot d_{k}$. Since $(i, j) \in S \subseteq F_{p}$ and $(i, k) \in F_{p}$, we have $u_{i, j}\left(p_{j}\right)=u_{i, k}\left(p_{k}\right)$ and, thus, $c_{i, j} \cdot d_{j}<c_{i, k} \cdot d_{k}$.

For the only if-part assume that $c_{i, j} \cdot d_{j} \leq c_{i, k} \cdot d_{k}$ for all $(i, j) \in E \subseteq F_{p}$ and all $(i, k) \in F_{p}$. Consider arbitrary edges $(i, j) \in E \subseteq F_{p}$ and $(i, k) \in F_{p}$. By piece-wise linearity, $u_{i, j}\left(p_{j}+\lambda \cdot d_{j}\right)=$ $u_{i, j}\left(p_{j}\right)-c_{i, j} \cdot \lambda \cdot d_{j}$ and $u_{i, k}\left(p_{k}+\lambda \cdot d_{k}\right)=u_{i, k}\left(p_{k}\right)-c_{i, k} \cdot \lambda \cdot d_{k}$ for all sufficiently small $\lambda>0.7$ Since $(i, j) \in E \subseteq F_{p}$ and $(i, k) \in F_{p}$, we have $u_{i, j}\left(p_{j}\right)=u_{i, k}\left(p_{k}\right)$. It follows that $u_{i, j}\left(p_{j}+\lambda \cdot d_{j}\right)=$ $u_{i, j}\left(p_{j}\right)-c_{i, j} \cdot \lambda \cdot d_{j} \geq u_{i, k}\left(p_{k}\right)-c_{i, k} \cdot \lambda \cdot d_{k}=u_{i, k}\left(p_{k}+\lambda \cdot d_{k}\right)$ for all sufficiently small $\lambda>0$, i.e., $d \neq 0$ is envy free for $E \subseteq F_{p}$.

Overdemand preserving price increase It is not difficult to see that envy free price increases are not enough to guarantee minimum prices. To achieve this goal we define a stronger notion of price increases, which exploits the correspondence between maximal alternating trees and strict overdemand: An overdemand preserving price increase $d$ for a maximal alternating tree $\mathcal{T}$ with respect to $\mu$ with root $i_{0}$ in $G_{p}$ with item set $S \subseteq J \backslash\left\{j_{0}\right\}$ and bidder set $T$ in which all items are matched, is a price increase $d$ such that (a) there is some partial matching $\mu^{\prime}$ that matches the same bidders and items as $\mu$ and that is identical to $\mu$ on $I \backslash T \times J \backslash S$, (b) there is a maximal alternating tree $\mathcal{T}^{\prime}$ with respect to $\mu^{\prime}$ with root $i_{0}$ that has the same item and bidder set as $\mathcal{T}$, and (c) $d$ is envy free for the edges of the maximal alternating tree $\mathcal{T}^{\prime}$. We say that $\mu^{\prime}$ is the matching that corresponds to $d$. Note that $\mu^{\prime}$ can be different from $\mu$ on $T \times S$. The crucial and new fact is that by (b) all items in the tree, i.e., all items whose price is increased, remain overdemanded for any small enough price increase.

Lemma 6. If $d$ is an overdemand preserving price increase for a maximal alternating tree $\mathcal{T}$ with respect to $\mu$ with root $i_{0}$ in $G_{p}$ with item set $S \subseteq J \backslash\left\{j_{0}\right\}$ and bidder set $T$ in which all items are matched, then $S$ is strictly overdemanded with respect to $T$ in $G_{p+\lambda \cdot d}$ for all sufficiently small $\lambda>0$.

Proof. Denote the partial matching and the maximal alternating tree corresponding to $d$ by $\mu^{\prime}$ and $\mathcal{T}^{\prime}$. Since $d$ is envy free for $\mathcal{T}^{\prime}$, Lemma 4 shows that all edges in $\mathcal{T}^{\prime}$ belong to the first choice graph $G_{p+\lambda \cdot d}$ at prices $p+\lambda \cdot d$ for all $\lambda>0$ sufficiently small. Since $\mathcal{T}^{\prime}$ is a maximal alternating tree with item set $S$ and bidder set $T$ in which all items are matched, Lemma 3 shows that (1) the set of items $S$ is strictly overdemanded with respect to the set of bidders $T$ and (2) $|T|=|S|+1$.

Next we present a subroutine that computes an overdemand preserving price increase $d$ and a corresponding matching $\mu^{\prime}$ for a maximal alternating tree $\mathcal{T}$ with respect to $\mu$ with root $i_{0}$ in $G_{p}$ with item set $S \subseteq J \backslash\left\{j_{0}\right\}$ and bidder set $T$ in which all items are matched. The computation consists of three steps: (1) The subroutine computes a matching $\sigma$ between $T \backslash\left\{i_{0}\right\}$ and $S$ consisting of first choice edges, which minimizes $\prod_{(i, j) \in \mu} c_{i, j}$, or equivalently, $\sum_{(i, j) \in \mu} \log \left(c_{i, j}\right)$. It also computes

\footnotetext{
${ }^{7}$ For this we need that the intervals are of the form [..).
} 
an envy free price increase $d$ for $\sigma$. This can be accomplished by solving a linear program (LP) and its dual (DP), e.g., by using a primal-dual algorithm [37]. The duality between slopes and utility drops exploited here is reminiscent of the duality between value-maximizing matchings and envy free prices in 45]. (2) The subroutine extends $d$ to an envy free direction for a maximal alternating tree $\mathcal{T}^{\prime}$ with respect to $\sigma$ with root $i_{0}$ in $G_{p}$ with bidder set $T$ and item set $S$. (3) The subroutine extends $\sigma$ to $\mu^{\prime}$ by adding to it the bidder-item pairs from $I \backslash T \times J \backslash S$ that were matched in $\mu$. While (1) is essentially an application of Lemma 5 (and has been used in a similar form in [3, 4]), (2) and (3) exploit the newly established correspondence between maximal alternating trees and strict overdemand.

PSEUDOCODE 1: Subroutine for price increases

Input: maximal alternating tree $\mathcal{T}$ with respect to $\mu$ with root $i_{0}$ in $G_{p}$ with item set $S$ and bidder set $T$ in which all items are matched

Output: overdemand preserving price increase $d$ for $\mathcal{T}$ with corresponding matching $\mu^{\prime}$ 1 compute $x$ as optimal solution to the following LP and let $\sigma=\left\{(i, j) \in T \backslash\left\{i_{0}\right\} \times S \mid x_{i, j}=1\right\}$

$$
\begin{array}{lll}
\min & \sum_{i, j} x_{i, j} \cdot \log \left(c_{i, j}\right) & \\
\mathrm{sb} & \sum_{j \in F_{p}(i)} x_{i, j}=1 & \left(\forall i \in T \backslash\left\{i_{0}\right\}\right) \\
& \sum_{i \in F_{p}(j)} x_{i, j}=1 & (\forall j \in S) \\
& x_{i, j} \geq 0 & \left(\forall(i, j) \in F_{p} \cap\left(T \backslash\left\{i_{0}\right\} \times S\right)\right)
\end{array}
$$

2 compute $\omega, \rho$ as optimal solution to the following DP

$$
\begin{array}{ll}
\max & \sum_{i} \omega_{i}+\sum_{j} \rho_{j} \\
\mathrm{sb} & \omega_{i}+\rho_{j} \leq \log \left(c_{i, j}\right) \quad\left(\forall(i, j) \in F_{p} \cap\left(T \backslash\left\{i_{0}\right\} \times S\right)\right)
\end{array}
$$

3 extend $\omega$ from $T \backslash\left\{i_{0}\right\}$ to $T$ by setting $\omega_{i_{0}}=\min _{j \in S} \log \left(c_{i_{0}, j}\right)-\rho_{j}$

4 let $H_{\rho}=\left(T \cup S, E_{\rho}\right)$, where $\quad E_{\rho}=\left\{(i, j) \in F_{\rho} \cap(T \times S) \mid \omega_{i}+\rho_{j}=\log \left(c_{i, j}\right)\right\}$

5 let $\mathcal{T}^{\prime}$ be a maximal alternating tree in $H_{\rho}$ with respect to $\sigma$ with root $i_{0}$

6 let $S^{\prime} \subseteq S$ and $T^{\prime} \subseteq T$ denote the items and bidders in $\mathcal{T}^{\prime}$

7 while $T^{\prime} \neq T$ or $S^{\prime} \neq S$ do

$8 \quad$ let $\delta=\min _{(i, j) \in F_{p}: i \in T^{\prime}, j \in S \backslash S^{\prime}} \log \left(c_{i, j}\right)-\omega_{i}-\rho_{j}$

$9 \quad$ set $\rho_{j}=\rho_{j}+\delta$ for all $j \in S \backslash S^{\prime}$, set $\omega_{i}=\omega_{i}-\delta$ for all $i \in T \backslash T^{\prime}$

10 recompute $\mathcal{T}^{\prime}, T^{\prime}$, and $S^{\prime}$

11 end

12 set $d_{j}=e^{-\rho_{j}}$ for all $j \in S$ and $d_{j}=0$ otherwise

13 set $\mu^{\prime}=\sigma \cup(\mu \cap(I \backslash T \times J \backslash S))$

14 output $d$ and $\mu^{\prime}$

Lemma 7. This subroutine finds an overdemand preserving price increase and a corresponding matching. It can be implemented to run in time $O\left(\min (n, k)^{3}\right)$.

Proof. Let $x, \omega$, and $\rho$ be defined as in the mechanism. The constraint matrix of LP is totally unimodular, i.e., $x_{i, j} \in\{0,1\}$ for all $(i, j) \in F_{p} \cap\left(T \backslash\left\{i_{0}\right\} \times S\right)$ [44. Hence $\sum_{j \in F_{p}(i)} x_{i, j}=1$ $\left(\forall i \in T \backslash\left\{i_{0}\right\}\right)$ and $\sum_{i \in F_{p}(j)} x_{i, j}=1(\forall j \in S)$ ensure that $\sigma=\left\{(i, j) \in T \backslash\left\{i_{0}\right\} \times S \mid x_{i, j}=1\right\}$ matches every bidder $i \in T \backslash\left\{i_{0}\right\}$ and every item $j \in S$ exactly once. From duality: 
1. For all $i \in T \backslash\left\{i_{0}\right\}$ and all $j \in S$ with $(i, j) \in \sigma: \omega_{i}+\rho_{j}=\log \left(c_{i, j}\right)$.

2. For all $i \in T \backslash\left\{i_{0}\right\}$ and all $j \in S: \omega_{i}+\rho_{j} \leq \log \left(c_{i, j}\right)$.

If we extend $\omega$ from $T \backslash\left\{i_{0}\right\}$ to $T$ as described in 1. 3 , then we also have:

3. There exists a $j \in S: \omega_{i_{0}}+\rho_{j}=\log _{c_{i_{0}, j}}$.

4. For all $j \in S: \omega_{i_{0}}+\rho_{j} \leq \log _{c_{i_{0}, j}}$.

Let $H_{\rho}=\left(S \cup T, E_{\rho}\right), E_{\rho}, \mathcal{T}^{\prime}, T^{\prime}$ and $S^{\prime}$ be defined as in 1l. 4-6. It is not difficult to see that the while-loop in 1l. 7-11 has the following properties:

a. For all $i \in T^{\prime}$ and all $j \in S^{\prime}$ : If we had $\omega_{i}+\rho_{j}<($ resp. $=) \log \left(c_{i, j}\right)$ before the update, then we have $\omega_{i}+\rho_{j}<($ resp. $=) \log \left(c_{i, j}\right)$ after the update.

b. For all $i \in T \backslash T^{\prime}$ and all $j \in S \backslash S^{\prime}$ : If we had $\omega_{i}+\rho_{j}<($ resp. $=) \log \left(c_{i, j}\right)$ before the update, then we have $\omega_{i}+\rho_{j}<$ (resp. $\left.=\right) \log \left(c_{i, j}\right)$ after the update.

c. For all $i \in T \backslash T^{\prime}$ and all $j \in S^{\prime}$ : If we had $\omega_{i}+\rho_{j} \leq \log \left(c_{i, j}\right)$ before the update, then we have $\omega_{i}+\rho_{j}<\log \left(c_{i, j}\right)$ after the update.

d. For all $i \in T^{\prime}$ and $j \in S \backslash S^{\prime}$ : If we had $\omega_{i}+\rho_{j}<\log \left(c_{i, j}\right)$ before the update, then we have $\omega_{i}+\rho_{j} \leq \log \left(c_{i, j}\right)$ after the update.

e. There exist at least one $i \in T^{\prime}$ and $j \in S \backslash S^{\prime}: \omega_{i}+\rho_{j}=\log \left(c_{i, j}\right)$ after the update.

From a. to e. we get that no edge from $\sigma$ and $\mathcal{T}^{\prime}$ in $E_{\rho}$ is lost. From e. we get that at least one edge from some $i \in T^{\prime}$ to some $j \in S \backslash S^{\prime}$ is added to $E_{\rho}$. Since this item $j$ was matched under $\sigma$ along an edge in $E_{\rho}$ to an item $i^{\prime} \in T \backslash T^{\prime}$, we know that after each iteration of the while-loop the maximal alternating tree $\mathcal{T}^{\prime}$ with respect to $\sigma$ with root $i_{0}$ in $H_{\rho}$ will at least cover the bidders and items in $T^{\prime} \cup\left\{i^{\prime}\right\}$ and $S^{\prime} \cup\{j\}$. Hence, after at most $|S| \leq k$ iterations, $\mathcal{T}^{\prime}$ will cover the same bidders and items as $\mathcal{T}$.

Let $d$ be defined as in 1. 12. Then $d \neq 0$ is envy free for $\mathcal{T}^{\prime}$ by Lemma 5 because:

1. For all $(i, j) \in \mathcal{T}^{\prime}$ and all $(i, k) \in F_{p}$ we have that $c_{i, j} \cdot d_{j} \leq c_{i, k} \cdot d_{k}$ because $e^{\omega_{i}}=e^{\log \left(c_{i, j}\right)-\rho_{j}}=$ $c_{i, j} \cdot e^{-\rho_{j}}=c_{i, j} \cdot d_{j}$ and $e^{\omega_{i}} \leq e^{\log \left(c_{i, k}\right)-\rho_{k}}=c_{i, k} \cdot e^{-\rho_{k}}=c_{i, k} \cdot d_{k}$.

2. For all $(i, j) \in \mu^{\prime} \backslash \mathcal{T}^{\prime}$ and $(i, k) \in F_{p}$ we trivially have that $c_{i, j} \cdot d_{j} \leq c_{i, k} \cdot d_{k}$ because $d_{j}=0$, $d_{k} \geq 0$, and $c_{i, k} \geq 0$.

Let $\mu^{\prime}$ be defined as in 1. 13, then $\mu^{\prime}$ matches the same bidders and items as $\mu$ because (1) $\mu^{\prime}$ is identical to $\sigma$ on $T \times S$ and (2) $\mu^{\prime}$ is identical to $\mu$ on $I \backslash T \times J \backslash S$.

The LP and the DP can be solved in time $O\left(\min (n, k)^{3}\right)$ [27, 46]. The maximal alternating tree $\mathcal{T}^{\prime}$ can be computed in time $O\left(\min (n, k)^{2}\right)$ using a breadth-first search approach. The while-loop in 1l. 8-12 can be implemented using "slack variables" $\delta_{j}=\min _{(i, j) \in F_{p}: i \in T^{\prime}}\left(\log \left(c_{i, j}\right)-\omega_{i}-\rho_{j}\right)$ for each item $j \in S \backslash S^{\prime}$ so that all iterations of the while loop take total time $O\left(\min (n, k)^{2}\right)$ : The initialization of the $\delta_{j}$ 's takes time $O\left(\min (n, k)^{2}\right)$ as for each of the up to $\min (n, k)$ items in $S \backslash S^{\prime}$ the minimum is computed over the up to $\min (n, k)$ bidders in $T^{\prime}$. In each iteration of the while-loop at least one bidder-item pair $(i, j) \in T^{\prime} \times S \backslash S^{\prime}$ is added to $T^{\prime} \times S^{\prime}$. Since $\left|S \backslash S^{\prime}\right| \leq \min (n, k)$ it follows that there are at most $\min (n, k)$ iterations. Using the $\delta_{j}$ 's for $j \in S \backslash S^{\prime}$ the $\delta$ in 1.8 can be computed in time $O(\min (n, k))$. When the $\omega_{i}$ 's and $\rho_{j}$ 's are updated in 1.9 , the $\delta_{j}$ 's are adapted in time $O(\min (n, k))$ by subtracting $\delta$ from each $\delta_{j}$. Thus, ll. 9 and 10 take time $O(\min (n, k))$ per iteration for a total of $O\left(\min (n, k)^{2}\right)$. Instead of re-computing the maximal alternating tree $\mathcal{T}^{\prime}$ in 1. 10 from scratch we can keep the old one and add the required edges. Thus maintaining $\mathcal{T}^{\prime}$ takes only time $O\left(\min (n, k)^{2}\right)$ for all iterations of the while loop. Additionally the $\delta_{j}$ 's must be updated. For each bidder that is added to $T^{\prime}$ all $\delta_{j}$ 's must be updated. This takes time $O(\min (n, k))$ per 
bidder. But this happens at most once for each of the up to $\min (n, k)$ bidders that are added to $T^{\prime}$, since no bidder is ever removed from $T^{\prime}$. Thus, all the updates to the $\delta_{j}$ 's that are required when bidders are added to $T^{\prime}$ take total time $O\left(\min (n, k)^{2}\right)$.

The following lemma - our key lemma and main technical improvement over [3, 4] - shows that if overdemand preserving price increases are used, then the resulting prices will be minimum over all envy free outcomes.

Lemma 8. Let $d$ be an overdemand preserving price increase for a maximal alternating tree $\mathcal{T}$ in $G_{p}$ with item set $S$ and bidder set $T$ in which all items are matched. Let $\lambda>0$ be the smallest scalar such that at $p+\lambda \cdot d$ (a) a bidder-item pair $(i, j) \in T \times J \backslash S$ enters $G_{p+\lambda \cdot d}$ or (b) the end point $e_{i, j}^{(t)}$ of some interval $t>0$ is reached. Then for any envy free outcome $\left(\mu^{\prime \prime}, p^{\prime \prime}\right)$ with $p^{\prime \prime} \geq p$ we have $p^{\prime \prime} \geq p+\lambda \cdot d$.

Proof. For a contradiction suppose that $p_{s}^{\prime \prime}<p_{s}+\lambda \cdot d_{s}$ for some $s \in S$. Choose $\epsilon>0$ such that $p_{s}^{\prime \prime}=p_{s}+(\lambda-\epsilon) \cdot d_{s}$. Note that $\epsilon \leq \lambda$ because $p_{s}^{\prime \prime} \geq p_{s}$. Let $A=\left\{j \in S \mid p_{j}^{\prime \prime}-p_{j} \leq(\lambda-\epsilon) \cdot d_{j}\right\}$ and let $B=F_{p+(\lambda-\epsilon) \cdot d}(A) \cap T$. Note that $A \neq \emptyset$ because $s \in A$.

Since $d$ is envy free for $\mathcal{T}^{\prime}$ and $p_{j} \leq p_{j}+(\lambda-\epsilon) \cdot d_{j}<p_{j}+\lambda \cdot d_{j} \leq e_{j}$ for all $j \in S$, i.e., for no $(i, j) \in T \times S$ there is a discontinuity in $u_{i, j}(\cdot)$ within the range $\left[p_{j}, p_{j}+(\lambda-\epsilon) \cdot d_{j}\right]$, we have $\mathcal{T}^{\prime} \subseteq F_{p+(\lambda-\epsilon) \cdot d}$. Since $\mathcal{T}^{\prime}$ covers all bidders in $T$ and items in $S$ and $A \subseteq S$ and $S$ is strictly overdemanded with respect to $T$, we have that $|B|=\left|F_{p+(\lambda-\epsilon) \cdot d}(A) \cap T\right|>|A|$.

Next we show that $|A| \geq\left|F_{p^{\prime \prime}}(B)\right|$. For this it suffices to show that $F_{p^{\prime \prime}}(i) \subseteq A$ for all $i \in B$. For a contradiction assume that there exists an $i \in B$ and a $k \notin A$ with $k \in F_{p^{\prime \prime}}(i)$. It follows that

$$
\begin{aligned}
& p_{k}^{\prime \prime}-p_{k}>(\lambda-\epsilon) \cdot d_{k}, \quad \text { and } \\
& u_{i, k}\left(p_{k}^{\prime \prime}\right) \geq u_{i, j}\left(p_{j}^{\prime \prime}\right) \quad \text { for all } j \text {. }
\end{aligned}
$$

But by the definition of $B$, and since $i \in B$, there must be a $j \in A$ such that $j \in F_{p+(\lambda-\epsilon) \cdot d}(i)$. It follows that

$$
\begin{aligned}
p_{j}^{\prime \prime}-p_{j} & \leq(\lambda-\epsilon) \cdot d_{j},
\end{aligned}
$$

Using the fact that the utility functions are strictly monotonically decreasing we get

$$
\begin{aligned}
u_{i, j}\left(p_{j}^{\prime \prime}\right) & \geq u_{i, j}\left(p_{j}+(\lambda-\epsilon) \cdot d_{j}\right) & & (\text { from (12) }) \\
& \geq u_{i, k}\left(p_{k}+(\lambda-\epsilon) \cdot d_{k}\right) & & (\text { from (13) }) \\
& >u_{i, k}\left(p_{k}^{\prime \prime}\right) . & & (\text { from (10) })
\end{aligned}
$$

Since this would give a contradiction to (11), we must have $F_{p^{\prime \prime}}(B) \subseteq A$, i.e., $|A| \geq\left|F_{p^{\prime \prime}}(B)\right|$. It follows that $|B|>|A| \geq F_{p^{\prime \prime}}(B)$. But this shows that in $\left(\mu^{\prime \prime}, p^{\prime \prime}\right)$ not all bidders can be matched in an envy free manner. Contradiction!

Bidder Optimal Outcome Our mechanism starts with an empty matching $\mu=\emptyset$ and prices $p=0$. It then matches one bidder after the other until eventually all bidders are matched. For this it computes a maximal alternating tree $\mathcal{T}$ with respect to $\mu$ with root $i_{0}$, where $i_{0}$ is the bidder to be matched, in the first choice graph $G_{p}$. If the alternating tree contains the dummy item $j_{0}$ or an unmatched item $j$, then by Lemma 3 the current matching $\mu$ can be augmented along an alternating path from $i_{0}$ to $j_{0}$ resp. $j$. If this is not the case, then - again by Lemma 3 - the items 
$S$ in the tree are strictly overdemanded with respect to the bidders $T$ in the tree. In this case the mechanism computes an overdemand preserving price increase $d$ together with a corresponding matching $\mu^{\prime}$ (using the subroutine from the previous subsection) and raises the prices in compliance with $d$ until (a) a bidder-item pair $(i, j) \in T \times J \backslash S$ enters the first choice graph $G_{p+\lambda \cdot d}$ or (b) the end point $e_{i, j}^{(t)}$ of some interval $t>0$ is reached. In either case the current matching $\mu$ is replaced with $\mu^{\prime}$ and the minimality of the new prices is guaranteed by Lemma 8 . If at least one of the new prices $p_{j}+\lambda \cdot d_{j}$ corresponds to a discontinuity, then one or multiple edges might drop out of the first choice graph. The mechanism corrects for this by removing such edges from the matching if necessary. If no discontinuity is reached, then the maximal alternating tree $\mathcal{T}$ rooted at $i_{0}$ grows by at least one item.

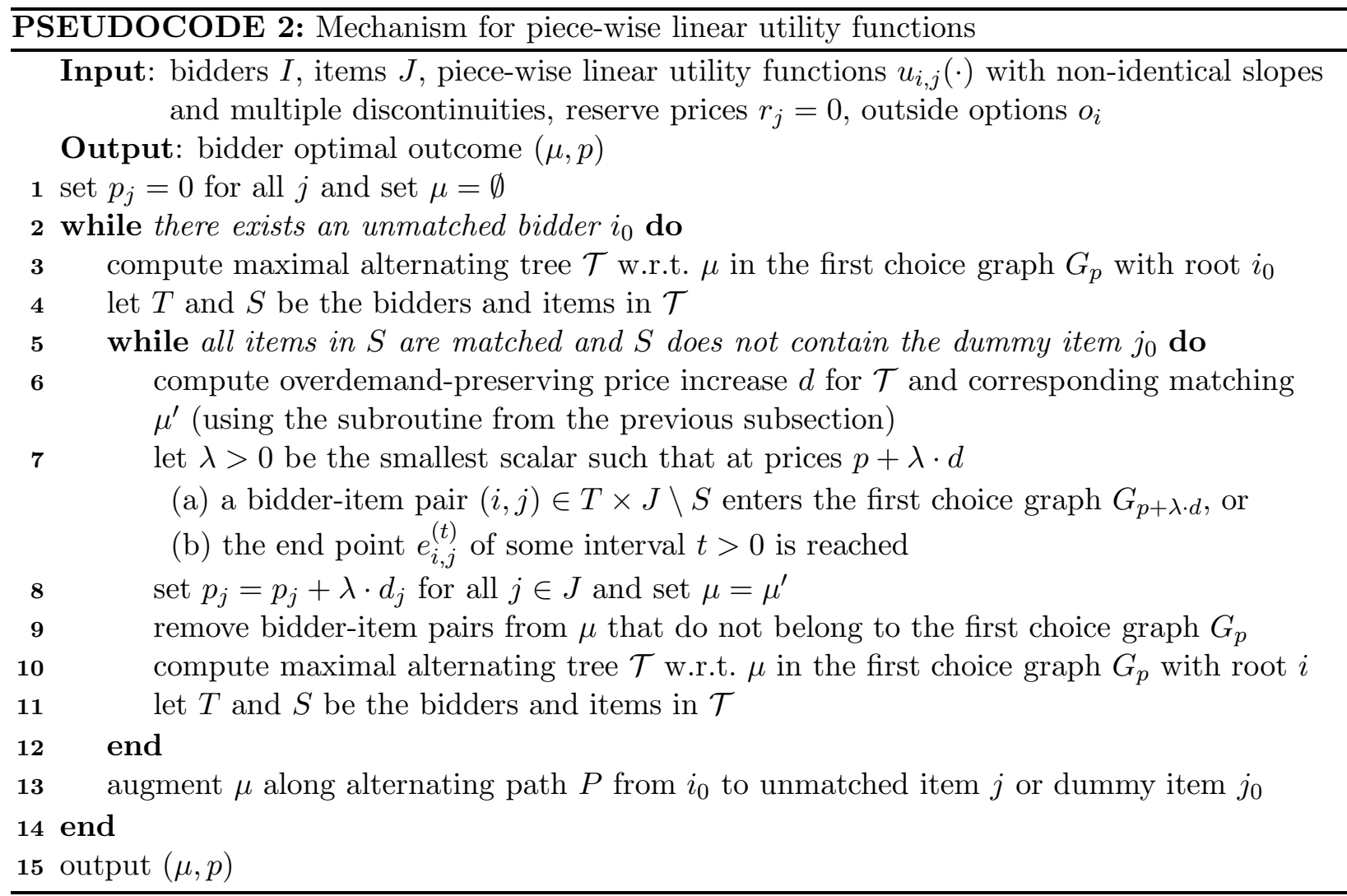

Theorem 1. This mechanism finds a bidder optimal outcome. It can be implemented to run in time $O\left((n \cdot \min (n, k)+D \cdot \min (n, k)+T) \cdot \min (n, k) \cdot\left(\min (n, k)^{2}+k\right)\right)$, where $D=\sum_{i, j} d_{i, j}$ and $T=\sum_{i, j} t_{i, j}$ denote the total number of discontinuities and constant-slope intervals.

Proof. The matching $\mu$ is a subset of the first choice edges at prices $p$. Hence Lemma 2 shows that $(\mu, p)$ is envy free. By Lemma 1 $(\mu, p)$ is bidder optimal if $p_{j} \leq p_{j}^{\prime \prime}$ for every item $j$ and every envy free outcome $\left(\mu^{\prime \prime}, p^{\prime \prime}\right)$. Let $p^{(t)}$ denote the prices after the $t$-th update. We prove that $p_{j}^{(t)} \leq p_{j}^{\prime \prime}$ for every item $j$, every envy free outcome $\left(\mu^{\prime \prime}, p^{\prime \prime}\right)$, and all time steps $t$ by induction over $t$.

$t=0$ : The claim follows from the fact that any envy free outcome $\left(\mu^{\prime \prime}, p^{\prime \prime}\right)$ has $p_{j}^{\prime \prime} \geq 0$ for all $j$.

$t-1 \rightarrow t$ : Assume that the claim is true for $t-1$. Let $\mathcal{T}$ be the maximal alternating tree with respect to the current matching $\mu^{(t-1)}$ with root $i_{0}$ right before the $t$-th update. Let $S$ and $T$ denote the items and bidders in $\mathcal{T}$. Let $d$ be an envy free price increase for $\mathcal{T}$ with corresponding 
alternating tree $\mathcal{T}^{\prime}$ and matching $\mu^{\prime}$. Let $\lambda$ be defined as in the mechanism. Note that $\lambda$ can be computed in time $O(\min (n, k) \cdot k$ by iterating over all bidders in $T$, of which there are at most $\min (n, k)$, and all items in $J$. The mechanism sets $p_{j}^{(t+1)}=p_{j}^{(t)}$ for $j \notin S$ and $p_{j}^{(t+1)}=p_{j}^{(t)}+\lambda \cdot d_{j}$ for $j \in S$. Lemma 8 shows that any envy free outcome $\left(\mu^{\prime \prime}, p^{\prime \prime}\right)$ with $p^{\prime \prime} \geq p^{(t)}$ must have $p_{j}^{\prime \prime} \geq p^{(t)}+\lambda \cdot d_{j}$. It follows that $p_{j}^{\prime \prime} \geq p_{j}^{(t+1)}$ for all $j$.

We bound the total time required by (1) the outer while-loop without the inner while-loop (ll. 24 \& 13-14) and the inner while-loop corresponding to Case (a) (1l. 5-12) separately from the total time required by (2) the inner while-loop corresponding to Case (b) (ll. 5-12).

To bound the total time required by (1) observe that: (i) In each iteration of the outer whileloop exactly one bidder gets matched. (ii) Bidders can get unmatched only if the boundary of a box is reached that corresponds to a discontinuity in at least one of the utility functions $u_{i, j}(\cdot)$. (iii) A discontinuity in $u_{i, j}(\cdot)$ can only unmatch bidder $i$. Since the prices are monotonically increasing at most $O\left(\sum_{i, j} d_{i, j}\right)$ discontinuities are reached. From (i) to (iii) we deduce that there are at most $O\left(n+\sum_{i, j} d_{i, j}\right)$ iterations of the outer-while loop without the inner while-loop and that the inner while-loop is executed at most $O\left(n+\sum_{i, j} d_{i, j}\right)$ times. Each iteration of the outer-while without the inner while-loop takes time $O\left(\min (n, k)^{2}\right)$. Each execution of the inner-while loop consists of at $\operatorname{most} \min (n, k)$ iterations corresponding to Case (a). Each of these iterations takes time $O\left(\min (n, k) \cdot\left(\min (n, k)^{2}+k\right)\right)$, namely $O\left(\min (n, k)^{3}\right)$ for computing the overdemand preserving price increase (see Lemma 7) and $O(\min (n, k) \cdot k)$ for computing the $\lambda$ value. Hence the total time required by (1) is $O\left(\left(n \cdot \min (n, k)+\sum_{i, j} d_{i, j} \cdot \min (n, k)\right) \cdot \min (n, k) \cdot\left(\min (n, k)^{2}+k\right)\right)$.

To bound the total time required by (2) observe that because the prices are monotonically increasing there are at most $O\left(\sum_{i, j} t_{i, j}\right)$ iterations of the inner-while loop that correspond to Case (b). As argued above each iteration of the inner while-loop takes time $O\left(\min (n, k) \cdot\left(\min (n, k)^{2}+k\right)\right)$. Hence the total time required by $(2)$ is $O\left(\left(\sum_{i, j} t_{i, j}\right) \cdot \min (n, k) \cdot\left(\min (n, k)^{2}+k\right)\right)$.

\section{Incentive Compatibility}

In this section we precisely characterize under which conditions any mechanism that computes a bidder optimal outcome is incentive compatible. Intuitively, a mechanism is incentive compatible if for every bidder $i$, independently of all other bidders, reporting his true utility functions yields an outcome, which gives him the highest possible utility. This can be formalized as follows: A mechanism is incentive compatible if for every bidder $i$ with utility functions $u_{i, j}(\cdot)$ and every two sets of utility functions $u_{i, j}^{\prime}(\cdot)$ and $u_{i, j}^{\prime \prime}(\cdot)$, where $u_{i, j}^{\prime}(\cdot)=u_{i, j}(\cdot)$ for $i$ and all $j$ and $u_{k, j}^{\prime}(\cdot)=u_{k, j}^{\prime \prime}(\cdot)$ for all $k \neq i$ and all $j$, and corresponding outcomes $\left(\mu^{\prime}, p^{\prime}\right)$ and $\left(\mu^{\prime \prime}, p^{\prime \prime}\right)$ of the mechanism we have

$$
u_{i, \mu^{\prime}(i)}\left(p_{\mu^{\prime}(i)}^{\prime}\right) \geq u_{i, \mu^{\prime \prime}(i)}\left(p_{\mu^{\prime \prime}(i)}^{\prime \prime}\right)
$$

Note that this definition does not involve the reserve prices $r_{j}$ or outside options $o_{i}$. This makes sense because the reserve prices $r_{j}$ are typically set by the seller and misreporting $o_{i}$ is never beneficial to $i 8$

Example: Lying pays off (This example shows that bidder optimality does not imply incentive compatibility.) There are two bidders $i \in\{1,2\}$ and two items $j \in\{1,2\}$. The utility functions for

\footnotetext{
${ }^{8}$ Over-reporting can only lead to a missed chance of being assigned an item and under-reporting can only lead to a utility below the true outside option.
} 
$i \in\{1,2\}$ are:

$$
\begin{aligned}
& u_{i, 1}\left(p_{1}\right)= \begin{cases}20-p_{1} & \text { for } p_{1} \in[0,5), \text { and } \\
-\infty & \text { otherwise }\end{cases} \\
& u_{i, 2}\left(p_{2}\right)=\quad 1-p_{2} \text { for } p_{2} \in[0, \infty) .
\end{aligned}
$$

The reserve prices are $r_{j}=0$ for $j \in\{1,2\}$ and the outside options are $o_{i}=0$ for $i \in\{1,2\}$. A bidder optimal outcome for this input is $\mu=\{(1,2)\}$ with $p_{1}=5$ and $p_{2}=1$. For this outcome both bidders have a utility of zero. Bidder 1 can improve his utility by pretending to have $u_{1,1}=0-p_{1}$ for $p_{1} \in[0, \infty)$. In this case the bidder optimal outcome is $\mu=\{(1,2),(2,1)\}$ with $p_{1}=p_{2}=0$. The utility of bidder 1 improves from 0 to 1 . The crucial point - as we will show below - is that in the computation of the bidder optimal outcome on this example two first choice edges, namely $(1,1)$ and $(2,1)$, simultaneously break away from the first choice graph at price $p_{1}=5$.

Price-Independent Formulation We will define next a condition on the input that implies that never during the execution of the mechanism two edges will break away from the first choice graph during the same price increase. Which edges break away depends on the current prices and the price increases. However, using the following idea we can write down a condition that does not depend on the current prices: Suppose that the edges $(i, j),\left(i^{\prime}, j\right)$, and $\left(i^{\prime}, j^{\prime}\right)$ belong to the first choice graph $G_{p}$ at prices $p$. It follows that

$$
v_{i^{\prime}, j}-c_{i^{\prime}, j} \cdot p_{j}=v_{i^{\prime}, j^{\prime}}-c_{i^{\prime}, j^{\prime}} \cdot p_{j^{\prime}} .
$$

Suppose further that $d$ is an envy free price increase for the set of first choice edges $E=\left\{(i, j),\left(i^{\prime}, j\right)\right.$, $\left.\left(i^{\prime}, j^{\prime}\right)\right\}$, then

$$
v_{i^{\prime}, j}-c_{i^{\prime}, j} \cdot\left(p_{j}+\lambda d_{j}\right)=v_{i^{\prime}, j^{\prime}}-c_{i^{\prime}, j^{\prime}} \cdot\left(p_{j^{\prime}}+\lambda d_{j^{\prime}}\right) .
$$

By subtracting (15) from (16), dividing by $\lambda>0$, and after rearranging we get

$$
d_{j}=c_{i^{\prime}, j^{\prime}} / c_{i, j} \cdot d_{j^{\prime}}
$$

Now suppose that the discontinuities $D_{i, j}$ and $D_{i^{\prime}, j^{\prime}}$ are reached simultaneously. Then by (16):

$$
v_{i^{\prime}, j}-c_{i^{\prime}, j} \cdot D_{i, j}=v_{i^{\prime}, j^{\prime}}-c_{i^{\prime}, j^{\prime}} \cdot D_{i^{\prime}, j^{\prime}} \cdot
$$

Using (17), subtracting $1 / d_{j} \cdot v_{i, j} / c_{i, j}$ from both sides, and after rearranging we get

$$
\frac{1}{d_{j}}\left(D_{i, j}-\frac{v_{i, j}}{c_{i, j}}\right)=-\frac{1}{d_{j}} \frac{v_{i, j}}{c_{i, j}}+\frac{1}{d_{j}} \frac{v_{i^{\prime}, j}}{c_{i^{\prime}, j}}+\frac{1}{d_{j^{\prime}}}\left(D_{i^{\prime}, j^{\prime}}-\frac{v_{i^{\prime}, j^{\prime}}}{c_{i^{\prime}, j^{\prime}}}\right) .
$$

Below we will define a multigraph such that the left and right hand side of this equation correspond to the weights of two alternating walks in the graph, namely $P=(i, j)$ and $Q=\left(i, j, i^{\prime}, j^{\prime}\right)$. Note that neither the weight of $P$ nor the weight of $Q$ depend on the prices.

General position For a given input we define a multigraph, called input graph, as follows: There is one node per bidder $i \in I$ and one node per item $j \in J$. There are three types of edges: (1) There is a forward edge from $i$ to $j$ for each linear segment of $u_{i, j}(\cdot)$. (2) There is a backward edge from $j$ to $i$ for each linear segment of $u_{i, j}(\cdot)$. (3) There is a discontinuity edge from $i$ to $j$ for each discontinuity $D_{i, j}$ of $u_{i, j}(\cdot)$. 
Let $P=\left(i_{0}, j_{1}, \ldots, i_{s}, j_{s}\right)$ be a walk in the input graph that alternates between forward and backward edges, and ends with a discontinuity edge. Let $d$ be a price increase such that $d_{j}=$ $\left(c_{i, j^{\prime}} / c_{i, j}\right) \cdot d_{j^{\prime}}$ for any two edges $(i, j)$ and $\left(i, j^{\prime}\right)$ on $P$. Define the weight of each forward edge $(i, j)$ on $P$ with respect to $d$ as $\left(-1 / d_{j}\right) \cdot\left(v_{i, j} / c_{i, j}\right)$, of each backward edge $(j, i)$ as $\left(1 / d_{j}\right) \cdot\left(v_{i, j} / c_{i, j}\right)$, and of the discontinuity edge $(i, j)$ as $\left(1 / d_{j}\right) \cdot\left(D_{i, j}-v_{i, j} / c_{i, j}\right)$. Here $v_{i, j}$ and $c_{i, j}$ are the constants of the corresponding linear segments. Define the weight $w_{d}(P)$ of $P$ with respect to $d$ as the sum of these weights. We say that the input is in general position if for no two walks $P$ and $Q$ that start with the same bidder and end with a distinct discontinuity edge and for no price increase $d$ such that $d_{j}=\left(c_{i, j^{\prime}} / c_{i, j}\right) \cdot d_{j^{\prime}}$ for any two edges $(i, j)$ and $\left(i, j^{\prime}\right)$ on $P$ resp. $Q$ we have $w_{d}(P)=w_{d}(Q)$. Note that this definition of general position is more general than that in [1]. In particular, it takes into account that the utility functions have non-identical slopes and multiple discontinuities.

Lemma 9. We have:

1. An input is in general position if and only if the associated input in standard form is in general position.

2. Let $(\mu, p)$ denote the outcome of the mechanism in Section [3. If the input is in general position, then

(i) no two discontinuities are reached simultaneously,

(ii) if an item gets unmatched, it gets matched again in the subsequent iteration,

(iii) if $p_{j}>r_{j}$, then item $j$ is matched under $\mu$, and

(iv) the last item, say $j$, that gets matched has $p_{j}=r_{j}$.

Proof. To 1.: Denote the original input by $\left(u_{i, j}(\cdot), r_{j}, o_{i}\right)$ and the associated input in standard form by $\left(u_{i, j}^{\prime}(\cdot), 0, o_{i}\right)$. Recall that $u_{i, j}^{\prime}\left(p_{j}\right)=u_{i, j}\left(p_{j}+r_{j}\right)$ for all $i$, all $j$, and all $p_{j} \geq 0$. Every pair of walks $P$ and $Q$ in the input graph for input $\left(u_{i, j}(\cdot), r_{j}, o_{i}\right)$ corresponds to a pair of walks $P^{\prime}$ and $Q^{\prime}$ in the input graph for input $\left(u_{i, j}^{\prime}(\cdot), 0, o_{i}\right)$, and vice versa. Since $c_{i, j}=c_{i, j}^{\prime}$ for every edge $(i, j)$ on $P$ and $P^{\prime}$ resp. $Q$ and $Q^{\prime}$, every price increase $d$ for which $d_{j}=\left(c_{i, j} / c_{i, j^{\prime}}\right) \cdot d_{j^{\prime}}$ for any two edges $(i, j)$ and $\left(i, j^{\prime}\right)$ on $P$ resp. $Q$ has $d_{j}=\left(c_{i, j}^{\prime} / c_{i, j^{\prime}}^{\prime}\right) \cdot d_{j^{\prime}}$ for any two edges $(i, j)$ and $\left(i, j^{\prime}\right)$ on $P^{\prime}$ resp. $Q^{\prime}$, and vice versa. Furthermore, since $v_{i, j}=v_{i, j}^{\prime}+c_{i, j} \cdot r_{j}$ for every edge $(i, j)$ on $P$ and $P^{\prime}$ resp. $Q$ and $Q^{\prime}$ and $D_{i, j}=D_{i, j}^{\prime}+r_{j}$ for every discontinuity edge $(i, j)$ on $P$ and $P^{\prime}$ resp. $Q$ and $Q^{\prime}$ (a) the weight of each forward edge $(i, j)$ is $-\left(1 / d_{j}\right) \cdot\left(v_{i, j} / c_{i, j}\right)=-\left(1 / d_{j}\right) \cdot\left(v_{i, j}^{\prime} / c_{i, j}\right)-\left(1 / d_{j}\right) \cdot r_{j}$, (b) the weight of each backward edge is $\left(1 / d_{j}\right) \cdot\left(v_{i, j} / c_{i, j}\right)=\left(1 / d_{j}\right) \cdot\left(v_{i, j}^{\prime} / c_{i, j}\right)+\left(1 / d_{j}\right) \cdot r_{j}$, and (c) the weight of each discontinuity edge is $\left(1 / d_{j}\right) \cdot D_{i, j}-\left(1 / d_{j}\right) \cdot\left(v_{i, j} / c_{i, j}\right)=\left(1 / d_{j}\right) \cdot D_{i, j}^{\prime}-\left(1 / d_{j}\right) \cdot\left(v_{i, j}^{\prime} / c_{i, j}\right)$. Since on each of the walks $P$ and $P^{\prime}$ resp. $Q$ and $Q^{\prime}$ there are as many forward as backward edges and every item $j$ is incident to exactly one forward and one backward edge, the $-\left(1 / d_{j}\right) \cdot r_{j}$ and $\left(1 / d_{j}\right) \cdot r_{j}$ terms cancel each other. Hence $w_{d}(P)$ is identical to $w_{d}\left(P^{\prime}\right)$ and $w_{d}(Q)$ is identical to $w_{d}\left(Q^{\prime}\right)$.

To 2.: We prove each of the claims (i) to (iv) individually.

To (i): For a contradiction suppose that two discontinuities are reached at the same time. W.l.o.g. assume that the discontinuities are reached on $\left(i_{1}, j_{1}\right)$ and $\left(i_{t}, j_{t-1}\right)$, and denote them by $D_{i_{1}, j_{1}}$ and $D_{i_{t}, j_{t-1}}$. Consider the walks $P=\left(i_{1}, j_{1}\right)$ and $Q=\left(i_{1}, j_{1}, i_{2}, j_{2}, \ldots, i_{t-1}, j_{t-1}, i_{t}, j_{t-1}\right)$, both alternate between forward and backward edges and end with a discontinuity edge. Note that these walks always exist because (1) either $j_{1}$ lies on the path from $i_{1}$ to $i_{t}$ or $i_{2}=i_{1}$ and (2) either $j_{t-1}$ lies on the path from $i_{1}$ to $i_{t}$ or $i_{t}=i_{t-1}$. Since the discontinuities are reached at the same time there must be a price increase $d \neq 0$ that is envy free for some maximal alternating tree containing $P$ and $Q$ and a positive scalar $\lambda>0$ such that $\lambda \cdot d_{j_{1}}=D_{i_{1}, j_{1}}-p_{j_{1}}$ and $\lambda \cdot d_{j_{t-1}}=D_{i_{t}, j_{t-1}}-p_{j_{t-1}}$. Rearranging this gives

$$
\frac{1}{d_{j_{1}}} \cdot D_{i_{1}, j_{1}}-\frac{1}{d_{j_{t-1}}} \cdot D_{i_{t}, j_{t-1}}=\frac{1}{d_{j_{1}}} \cdot p_{j_{1}}-\frac{1}{d_{j_{t-1}}} \cdot p_{j_{t-1}}
$$


From the fact that $P$ and $Q$ belong to the first choice graph $G_{p}$ at prices $p$ we get that for $s=1$..t-2 : $v_{i_{s+1}, j_{s}}-c_{i_{s+1}, j_{s}} \cdot p_{j_{s}}=v_{i_{s+1}, j_{s+1}}-c_{i_{s+1}, j_{s+1}} \cdot p_{j_{s+1}}(\star)$. Solving for $p_{j_{s}}$ we get that for $s=1 . . t-2$ :

$$
p_{j_{s}}=\frac{v_{i_{s+1}, j_{s}}}{c_{i_{s+1}, j_{s}}}-\frac{v_{i_{s+1}, j_{s+1}}}{c_{i_{s+1}, j_{s}}}+\frac{c_{i_{s+1}, j_{s+1}}}{c_{i_{s+1}, j_{s}}} \cdot p_{j_{s+1}} .
$$

From the fact that $P$ and $Q$ also belong to the first choice graph $G_{p+\lambda \cdot d}$ at prices $p+\lambda \cdot d$ we get that for $s=1 . . t-2: v_{i_{s+1}, j_{s}}-c_{i_{s+1}, j_{s}} \cdot\left(p_{j_{s}}+\lambda \cdot d_{j_{s}}\right)=v_{i_{s+1}, j_{s+1}}-c_{i_{s+1}, j_{s+1}} \cdot\left(p_{j_{s+1}}+\lambda \cdot d_{j_{s+1}}\right)$. Subtracting $(\star)$ and solving for $d_{j_{s+1}}$ we get that for $s=1 . . t-2$ :

$$
d_{j_{s+1}}=\frac{c_{i_{s+1}, j_{s}}}{c_{i_{s+1}, j_{s+1}}} \cdot d_{j_{s}}
$$

Solving the recurrence (20) for $p_{j_{1}}$, substituting (21), and rearranging gives:

$$
\frac{1}{d_{j_{1}}} \cdot p_{j_{1}}-\frac{1}{d_{j_{t-1}}} \cdot p_{j_{t-1}}=\frac{1}{d_{j_{2}}} \cdot \frac{v_{i_{2}, j_{1}}}{c_{i_{2}, j_{2}}}-\frac{1}{d_{j_{2}}} \cdot \frac{v_{i_{2}, j_{2}}}{c_{i_{2}, j_{2}}}+\cdots+\frac{1}{d_{j_{t-1}}} \cdot \frac{v_{i_{t-1}, j_{t-2}}}{c_{i_{t-1}, j_{t-1}}}-\frac{1}{d_{j_{t-1}}} \cdot \frac{v_{i_{t-1}, j_{t-1}}}{c_{i_{t-1}, j_{t-1}}} .
$$

We combine this with (19) to get

$$
\frac{1}{d_{j_{1}}} \cdot D_{i_{1}, j_{1}}-\frac{1}{d_{j_{t-1}}} \cdot D_{i_{t}, j_{t-1}}=\frac{1}{d_{j_{2}}} \cdot \frac{v_{i_{2}, j_{1}}}{c_{i_{2}, j_{2}}}-\frac{1}{d_{j_{2}}} \cdot \frac{v_{i_{2}, j_{2}}}{c_{i_{2}, j_{2}}}+\cdots+\frac{1}{d_{j_{t-1}}} \cdot \frac{v_{i_{t-1}, j_{t-2}}}{c_{i_{t-1}, j_{t-1}}}-\frac{1}{d_{j_{t-1}}} \cdot \frac{v_{i_{t-1}, j_{t-1}}}{c_{i_{t-1}, j_{t-1}}} .
$$

We add $\left(-1 / d_{j_{1}}\right) \cdot\left(v_{i_{1}, j_{1}} / c_{i_{1}, j_{1}}\right)$ to both sides and $\left(1 / d_{j_{t}}\right) \cdot\left(v_{i_{t}, j_{t-1}} / c_{i_{t}, j_{t}}\right)-\left(1 / d_{j_{t-1}}\right) \cdot\left(v_{i_{t}, j_{t-1}} / c_{i_{t}, j_{t-1}}\right)=$ 0 to the right hand side. After rearranging we get:

$$
\begin{aligned}
\frac{1}{d_{j_{1}}} \cdot D_{i_{1}, j_{1}}-\frac{1}{d_{j_{1}}} \cdot \frac{v_{i_{1}, j_{1}}}{c_{i_{1}, j_{1}}}= & -\frac{1}{d_{j_{1}}} \cdot \frac{v_{i_{1}, j_{1}}}{c_{i_{1}, j_{1}}}+\frac{1}{d_{j_{2}}} \cdot \frac{v_{i_{2}, j_{1}}}{c_{i_{2}, j_{2}}}-\frac{1}{d_{j_{2}}} \cdot \frac{v_{i_{2}, j_{2}}}{c_{i_{2}, j_{2}}}+\cdots+\frac{1}{d_{j_{t-1}}} \cdot \frac{v_{i_{t-1}, j_{t-2}}}{c_{i_{t-1}, j_{t-1}}} \\
& -\frac{1}{d_{j_{t-1}}} \cdot \frac{v_{i_{t-1}, j_{t-1}}}{c_{i_{t-1}, j_{t-1}}}+\frac{1}{d_{j_{t}}} \cdot \frac{v_{i_{t}, j_{t-1}}}{c_{i_{t}, j_{t}}}+\frac{1}{d_{j_{t-1}}} \cdot\left(D_{i_{t}, j_{t-1}}-\frac{v_{i_{t}, j_{t-1}}}{c_{i_{t}, j_{t-1}}}\right) .
\end{aligned}
$$

Since the left hand side corresponds to $w_{d}(P)$ and the right hand side to $w_{d}(Q)$, we get a contradiction to the fact that the input is in general position.

To (ii): Consider an arbitrary price update. Let $i_{0}$ be the bidder, and let $\mu$ be the matching under consideration. Denote the maximal alternating tree with respect to $\mu$ with root $i_{0}$ by $\mathcal{T}$. Let $d$ be the overdemand preserving price increase for $\mathcal{T}$ computed by our our mechanism. Denote the corresponding maximal alternating tree and matching by $\mathcal{T}^{\prime}$ resp. $\mu^{\prime}$. We know that $\mu^{\prime}$ matches the same set of bidders and items as $\mu$. If the price update corresponds to a $\lambda_{\text {out }}<\lambda_{\text {box }}$, then none of the edges in $\mathcal{T}^{\prime}$ drops out of the first choice graph $G_{p+\lambda \cdot d}$, i.e., none of the edges has to be removed from $\mu^{\prime}$. If it corresponds to a $\lambda_{\text {box }} \geq \lambda_{\text {out }}$, then - due to the fact that the input is in general position - at most one edge in $\mathcal{T}^{\prime}$ drops out of the first choice graph $G_{p+\lambda \cdot d}$. If the edge is an unmatched edge, then no edge has to be removed from $\mu^{\prime}$. If it is a matched edge, then exactly one edge has to be removed from $\mu^{\prime}$. But since all items in $\mathcal{T}^{\prime}$ are closer to the root $i_{0}$ than the bidder they are matched to, there must be an alternating path $P$ from the unmatched item, say $j$, to the root $i_{0}$. It follows that in the subsequent iteration there is exactly one alternating path along which the matching can be augmented, namely the path from $i_{0}$ to $j$. Hence $j$ gets matched again in the subsequent iteration.

To (iii): Our mechanism raises the price of an item strictly above its reserve price only if this item belonged to the set of items $S$ in a maximal alternating tree $\mathcal{T}$ in which all items are matched. From (i) we know that every item that ever got matched, will be matched in the end.

To (iv): Consider the iteration in which the last item $j$ gets matched. Since $j$ belongs to $F_{p}(T)$, we must have $p_{j} \geq r_{j}$. From (i) we know that item $j$ was never matched before and from (ii) we know that $p_{j}>r_{j}$ would imply that $j$ was matched before. Thus we must have $p_{j}=r_{j}$. 
Characterization We already know that bidder optimality does not imply incentive compatibility, if the input is not in general position. With the help of the following lemma - which is a generalization of Hwang's Lemma (see, e.g., [29, 20]) - we can show that any mechanism that computes a bidder optimal outcome is incentive compatible if conditions (iii) and (iv) from Lemma 9 are satisfied. An easy corollary is that if the input is in general position, then any mechanism that computes a bidder optimal outcome is incentive compatible. Note that while we do not have a polynomial-time algorithm to check whether an input is in general position, we can easily check whether conditions (iii) and (iv) are satisfied using our mechanism from Section 3 .

Lemma 10. If conditions (iii) and (iv) from Lemma 9 are satisfied, then:

1. If the outcome $\left(\mu^{*}, p^{*}\right)$ is bidder optimal, then for no feasible outcome $\left(\mu^{\prime}, p^{\prime}\right)$ we can have $u_{i, \mu^{\prime}(i)}\left(p_{\mu^{\prime}(i)}^{\prime}\right)>u_{i, \mu^{*}(i)}\left(p_{\mu^{*}(i)}^{*}\right)$ for all $i$.

2. If the outcome $\left(\mu^{*}, p^{*}\right)$ is bidder optimal, the outcome $\left(\mu^{\prime}, p^{\prime}\right)$ is feasible, and $I^{+} \neq \emptyset$, where $I^{+}=\left\{i \in I \mid u_{i, \mu^{\prime}(i)}\left(p_{\mu^{\prime}(i)}^{\prime}\right)>u_{i, \mu^{*}(i)}\left(p_{\mu^{*}(i)}^{*}\right)\right\}$, then there exists a bidder-item pair $(i, j) \in$ $I \backslash I^{+} \times J$ such that $u_{i, \mu^{\prime}(i)}\left(p_{\mu^{\prime}(i)}^{\prime}\right)<u_{i, j}\left(p_{j}^{\prime}\right)$.

Proof. To 1.: For a contradiction assume that there is a feasible outcome $\left(\mu^{\prime}, p^{\prime}\right)$ with $u_{i, \mu^{\prime}(i)}\left(p_{\mu^{\prime}(i)}^{\prime}\right)>$ $u_{i, \mu^{*}(i)}\left(p_{\mu^{*}(i)}^{*}\right)$ for all $i$. Let $(\mu, p)$ denote the outcome of our mechanism. Since $u_{i, \mu(i)}\left(p_{\mu(i)}\right)=$ $u_{i, \mu^{*}(i)}\left(p_{\mu^{*}(i)}^{*}\right)$ for all $i$, it follows that $u_{i, \mu^{\prime}(i)}\left(p_{\mu^{\prime}(i)}^{\prime}\right)>u_{i, \mu(i)}\left(p_{\mu(i)}\right)$ for all $i$.

Consider any pair $(i, j) \in \mu^{\prime}$. It follows that $u_{i, j}\left(p_{j}^{\prime}\right)=u_{i, \mu^{\prime}(i)}\left(p_{\mu^{\prime}(i)}^{\prime}\right)>u_{i, \mu(i)}\left(p_{\mu(i)}\right) \geq u_{i, j}\left(p_{j}\right)$ and, thus, $p_{j}>p_{j}^{\prime} \geq r_{j}$. Condition (iii) from Lemma 9 implies that item $j$ is matched under $\mu$. We conclude that (1) all the items that are matched under $\mu^{\prime}$ are also matched under $\mu$ and (2) $p_{j}^{\prime}<p_{j}$ for all of these items $j$.

Case 1: At least one bidder $i$ is matched to the dummy item $j_{0}$ under $\mu^{\prime}$. By $(2) p_{j_{0}}>p_{j_{0}}^{\prime} \geq 0$, which contradicts the feasibility of the outcome $(\mu, p)$.

Case 2: All bidders $i$ are matched to non-dummy items $j$ under $\mu^{\prime}$. By (1) all bidders are matched to non-dummy items under $\mu$. Condition (iv) from Lemma 9 shows that at least one item $j$ is matched under $\mu$ at price $p_{j}=r_{j}$. By (2) $p_{j}^{\prime}<p_{j}=r_{j}$, which contradicts the feasibility of the outcome $\left(\mu^{\prime}, p^{\prime}\right)$.

To 2.: Let $(\mu, p)$ denote the outcome of our mechanism. Since $u_{i, \mu(i)}\left(p_{\mu(i)}\right)=u_{i, \mu^{*}(i)}\left(p_{\mu^{*}(i)}^{*}\right)$ for all $i$, we have $I^{+}=\left\{i \in I \mid u_{i, \mu^{\prime}(i)}\left(p_{\mu^{\prime}(i)}^{\prime}\right)>u_{i, \mu(i)}\left(p_{\mu(i)}\right)\right\} \neq \emptyset$. Let $\mu\left(I^{+}\right)$resp. $\mu^{\prime}\left(I^{+}\right)$denote the set of items matched to bidders in $I^{+}$under $\mu$ resp. $\mu^{\prime}$. From the first part of this lemma we know that $I^{+} \neq I$.

Case 1: $\mu\left(I^{+}\right) \neq \mu^{\prime}\left(I^{+}\right)$. There must be an item $j \in \mu^{\prime}\left(I^{+}\right)$such that $j \notin \mu\left(I^{+}\right)$. Let $i^{\prime} \in I^{+}$ be the bidder that is matched to item $j$ in $\mu^{\prime}$. Since $i^{\prime} \in I^{+}$and the outcome $(\mu, p)$ is envy free we have that $u_{i^{\prime}, j}\left(p_{j}^{\prime}\right)=u_{i^{\prime}, \mu^{\prime}\left(i^{\prime}\right)}\left(p_{\mu^{\prime}\left(i^{\prime}\right)}^{\prime}\right)>u_{i^{\prime}, \mu\left(i^{\prime}\right)}\left(p_{\mu\left(i^{\prime}\right)}\right) \geq u_{i^{\prime}, \mu^{\prime}\left(i^{\prime}\right)}\left(p_{\mu^{\prime}\left(i^{\prime}\right)}\right)=u_{i^{\prime}, j}\left(p_{j}\right)$ which shows that $p_{j}>p_{j}^{\prime}$. Hence $j$ is matched under $\mu$ by condition (iii) from Lemma 9, Let $i \in I \backslash I^{+}$be the bidder that is matched to item $j$ under $\mu$. Since $i \notin I^{+}$it follows that $u_{i, \mu^{\prime}(i)}\left(p_{\mu^{\prime}(i)}^{\prime}\right) \leq u_{i, \mu(i)}\left(p_{\mu(i)}\right)=$ $u_{i, j}\left(p_{j}\right)<u_{i, j}\left(p_{j}^{\prime}\right)$.

Case 2: $\mu\left(I^{+}\right)=\mu^{\prime}\left(I^{+}\right)$. Let $J^{+}=\mu\left(I^{+}\right)=\mu^{\prime}\left(I^{+}\right)$. Consider the following restricted problem: The set of bidders is $I^{+}$, the set of items is $J^{+}$, the utility functions are $u_{i, j}^{+}(\cdot)=u_{i, j}(\cdot)$ for all $(i, j) \in I^{+} \times J^{+}$, the reserve prices are $r_{j}^{+}=\max \left(r_{j}, \max _{i \notin I^{+}}\left(u_{i, j}^{-1}\left(u_{i, \mu(i)}\left(p_{\mu(i)}\right)\right), 0\right)\right)$ for all $j \in J^{+}$, and the outside options are $o_{i}^{+}=o_{i}$ for all $i \in I^{+}$. 9 Since the outcome $(\mu, p)$ is envy free for the

\footnotetext{
${ }^{9}$ If $u_{i, j}(\cdot)$ is continuous then $u_{i, j}^{-1}(\cdot)$ is indeed the inverse function. More generally, it is defined for $u \in\left[o_{i}, \infty\right)$ by $u_{i, j}^{-1}(u):=\min _{p_{j} \in\left[r_{j}, \infty\right)}\left\{u_{i, j}\left(p_{j}\right) \leq u\right\}$, and is merely a one-sided inverse function satisfying $u_{i, j}^{-1}\left(u_{i, j}\left(p_{j}\right)\right)=p_{j}$. Note that the minimum is indeed contained in the set itself as we only consider right-continuous utility functions.
} 
original input it is also envy free for the restricted input. It is even bidder optimal because the existence of an envy free outcome $\left(\mu^{\prime \prime}, p^{\prime \prime}\right)$ for the restricted problem in which at least one bidder $i \in I^{+}$has a strictly higher utility would imply the existence of an envy free outcome $\left(\mu^{\prime \prime \prime}, p^{\prime \prime \prime}\right)$ for the original problem with this property and therefore contradict the bidder optimality of $(\mu, p)$.

Case 2.1: The outcome $\left(\mu^{\prime}, p^{\prime}\right)$ is feasible for the restricted problem. From the first part of this lemma we know that there exists a bidder $i \in I^{+}$such that $u_{i, \mu^{\prime}(i)}\left(p_{\mu^{\prime}(i)}^{\prime}\right) \leq u_{i, \mu(i)}\left(p_{\mu(i)}\right)$. This contradicts the definition of $I^{+}$.

Case 2.2: The outcome $\left(\mu^{\prime}, p^{\prime}\right)$ is not feasible for the restricted problem. This can only happen if $p_{j}^{\prime}<r_{j}^{+}$for some item $j \in J^{+}$. Since the outcome $\left(\mu^{\prime}, p^{\prime}\right)$ is feasible for the original problem this can only happen if $r_{j}^{+}>r_{j}$ and so $r_{j}^{+}=\max _{i \notin I^{+}}\left(u_{i, j}^{-1}\left(u_{i, \mu(i)}\left(p_{\mu(i)}\right)\right), 0\right)$. We cannot have $r_{j}^{+}=0$ as this would imply $p_{j}^{\prime}<r_{j}^{+}=0$. Thus we must have $r_{j}^{+}=u_{i, j}^{-1}\left(u_{i, \mu(i)}\left(p_{\mu(i)}\right)\right)$ for some $i \in I \backslash I^{+}$. It follows that $p_{j}^{\prime}<r_{j}^{+}=u_{i, j}^{-1}\left(u_{i, \mu(i)}\left(p_{\mu(i)}\right)\right) \leq u_{i, j}^{-1}\left(u_{i, \mu^{\prime}(i)}\left(p_{\mu^{\prime}(i)}^{\prime}\right)\right)$ and so $u_{i, \mu^{\prime}(i)}\left(p_{\mu^{\prime}(i)}^{\prime}\right)<u_{i, j}\left(p_{j}^{\prime}\right)$.

Theorem 2. If conditions (iii) and (iv) from Lemma 9] are satisfied, then any mechanism that computes a bidder optimal is incentive compatible.

Proof. For a contradiction suppose some subset of bidders $I^{+} \subseteq I$ strictly benefits from misreporting their utility functions. Denote the true input by $\left(u_{i, j}(\cdot), r_{j}, o_{i}\right)$, and the falsified one by $\left.\left(u_{i, j}^{\prime}(\cdot)\right), r_{j}, o_{i}\right)$. Note that $u_{i, j}^{\prime}(\cdot)=u_{i, j}(\cdot)$ for all $(i, j) \in I \backslash I^{+} \times J$. Let $\left(\mu^{*}, p^{*}\right)$ resp. $\left(\mu^{\prime}, p^{\prime}\right)$ denote the bidder optimal outcome for the true resp. falsified input. Then $I^{+}=\left\{i \in I \mid u_{i, \mu^{\prime}(i)}\left(p_{\mu^{\prime}(i)}^{\prime}\right)>\right.$ $\left.u_{i, \mu^{*}(i)}\left(p_{\mu^{*}(i)}^{*}\right)\right\}$. Note that $\left(\mu^{\prime}, p^{\prime}\right)$ is feasible for the true input $\left(u_{i, j}(\cdot), r_{j}, o_{i}\right)$ because $p_{j_{0}}^{\prime}=0$ and $p_{j}^{\prime} \geq 0$ for all $j \neq j_{0}$.

Case 1: $I^{+}=I$. The first part of Lemma 10 shows that if condition (iii) and (iv) from Lemma 9 are satisfied, then no feasible outcome $\left(\mu^{\prime}, p^{\prime}\right)$ can give all bidders a strictly higher utility than the bidder optimal outcome $\left(\mu^{*}, p^{*}\right)$. This gives a contradiction.

Case 2: $I^{+} \neq I$. The second part of Lemma 10 shows that if condition (iii) and (iv) from Lemma 9 are satisfied, then some feasible outcome $\left(\mu^{\prime}, p^{\prime}\right)$ gives only some of the bidders a strictly higher utility than the bidder optimal outcome $\left(\mu^{*}, p^{*}\right)$, then there must be at least one bidder $i \in I \backslash I^{+}$and an item $j \in S$ for which $u_{i, \mu^{\prime}(i)}\left(p_{\mu^{\prime}(i)}^{\prime}\right)<u_{i, j}\left(p_{j}^{\prime}\right)$. But since $i \notin I^{+}$, i.e., $i$ is not among the liars, this implies that $u_{i, \mu^{\prime}(i)}^{\prime}\left(p_{\mu^{\prime}(i)}^{\prime}\right)=u_{i, \mu^{\prime}(i)}\left(p_{\mu^{\prime}(i)}^{\prime}\right)<u_{i, j}\left(p_{j}^{\prime}\right)=u_{i, j}^{\prime}\left(p_{j}^{\prime}\right)$ and contradicts the fact that $\left(\mu^{\prime}, p^{\prime}\right)$ is bidder optimal and therefore envy free for the falsified input $\left.\left(u_{i, j}^{\prime}(\cdot)\right), r_{j}, o_{i}\right)$.

We conclude our discussion of the incentives involved in computing bidder optimal outcomes with several interesting open questions concerning the general position concept: First, what does it take (time-wise) to evaluate whether an input is in general position? Second, what does it take to change an input that is not in general position to be in general position? Finally, under which conditions is a bidder optimal solution to an input that has been brought into general position also bidder optimal for the original input?

\section{Approximation}

In this section we show how our mechanism for piece-wise linear utility functions can be applied to compute approximately bidder optimal outcomes for a general class of continuous utility functions $u_{i, j}(\cdot)$. The idea is as follows: Approximate each utility function $u_{i, j}(\cdot)$ by a piece-wise linear utility function $\tilde{u}_{i, j}(\cdot)$. Then solve the problem for this approximated input exactly and use the outcome $(\tilde{\mu}, \tilde{p})$ obtained as an approximate solution to the original problem. 
More specifically, we say that an outcome $(\tilde{\mu}, \tilde{p})$ is $\gamma$-envy free for the input $u_{i, j}(\cdot)$ if it is feasible and $u_{i, \tilde{\mu}(i)}\left(\tilde{p}_{\tilde{\mu}(i)}\right)+\gamma \geq u_{i, j}\left(\tilde{p}_{j}\right)$ for all $(i, j) \in I \times J$. An outcome $(\tilde{\mu}, \tilde{p})$ is $\gamma$-bidder optimal if it is $\gamma$-envy free and for any envy free outcome $\left(\tilde{\mu}^{\prime}, \tilde{p}^{\prime}\right)$ we have $u_{i, \tilde{\mu}(i)}\left(\tilde{p}_{\tilde{\mu}(i)}\right)+\gamma \geq u_{i, \tilde{\mu}^{\prime}(i)}\left(\tilde{p}_{\tilde{\mu}^{\prime}(i)}^{\prime}\right)$.

Apart from the assumptions that the utility functions $u_{i, j}(\cdot)$ are strictly monotonically decreasing and continuous we make the following mild assumptions concerning the first and second derivatives $\dot{u}_{i, j}(\cdot)$ and $\ddot{u}_{i, j}(\cdot)$ of $u_{i, j}(\cdot)$, which allow us to bound the number of linear segments needed for piece-wise linear approximation:

(A.1) The utility functions $u_{i, j}(\cdot)$ are twice differentiable on $\left[r_{j}, \bar{p}_{i, j}\right]$, i.e., $\ddot{u}_{i, j}\left(p_{j}\right)$ exists on this interval.

(A.2) There exists a constant $B$ such that $\forall(i, j) \in I \times J: \max _{p_{j} \in\left[r_{j}, \bar{p}_{i, j}\right]}\left|\ddot{u}_{i, j}\left(p_{j}\right)\right| \leq B$.

(A.3) There exist constants $m$ and $M$ such that $\forall(i, j) \in I \times J$ and $\forall p_{j} \in\left[r_{j}, \bar{p}_{i, j}\right]: 0<m \leq$ $\left|\dot{u}_{i, j}\left(p_{j}\right)\right| \leq M$.

Note that we use $\dot{u}_{i, j}(\cdot)$ and $\ddot{u}_{i, j}(\cdot)$ to denote the first and second derivative of $u_{i, j}(\cdot)$ to avoid confusion with $u_{i, j}^{\prime}(\cdot)$ and $u_{i, j}^{\prime \prime}(\cdot)$, which were previously used in a different context.

Piece-wise linear approximation Given $\epsilon>0$ we construct a piece-wise linear, continuous approximation $\tilde{u}_{i, j}(\cdot)$ for $u_{i, j}(\cdot)$ with error at most $\epsilon$, i.e., $\left|u_{i, j}\left(p_{j}\right)-\tilde{u}_{i, j}\left(p_{j}\right)\right| \leq \epsilon$ for all $p_{j} \epsilon$ $\left[r_{j}, \bar{p}_{i, j}\right]$, as follows: First, as $u_{i, j}(\cdot)$ can extend from $r_{j}$ to $\infty$ and could potentially require an infinite number of segments to approximate, we limit the approximation to the range $\left[r_{j}, \bar{p}_{i, j}\right]$ as follows: Since $p_{j}>\bar{p}_{i, j}$ cannot correspond to a match as then $u_{i, j}\left(p_{j}\right)<o_{i}=u_{i, j_{0}}\left(p_{j_{0}}\right)$, we can extend $u_{i, j}\left(p_{j}\right)$ for prices $p_{j}>\bar{p}_{i, j}$ in a continuous and differentiable way by the line $\tilde{u}_{i, j}\left(p_{j}\right)=$ $\dot{u}_{i, j}\left(\bar{p}_{i, j}\right) \cdot p_{j}-\dot{u}_{i, j}\left(\bar{p}_{i, j}\right) \cdot \bar{p}_{i, j}$. This limits the "interesting" domain to $\left[r_{j}, \bar{p}_{i, j}\right]$. Next we split the range $\left[r_{j}, \bar{p}_{i, j}\right]$ into $S=\left\lceil\left(\bar{p}_{i, j}-r_{j}\right) / \sqrt{8 \epsilon / B}\right\rceil$ intervals of equal width $w=\left(\bar{p}_{i, j}-r_{j}\right) / S$. On any interval with endpoints $\left[\tilde{e}^{(t)}, \tilde{e}^{(t+1)}\right]$, where $\tilde{e}^{(t+1)}=\tilde{e}^{(t)}+w$, the line $\tilde{u}_{i, j}(\cdot)$ used to approximate $u_{i, j}(\cdot)$ is defined by

$$
\tilde{u}_{i, j}\left(p_{j}\right)=\frac{u_{i, j}\left(\tilde{e}^{(t+1)}\right)-u_{i, j}\left(\tilde{e}^{(t)}\right)}{\tilde{e}^{(t+1)}-\tilde{e}^{(t)}} p_{j}+\frac{u_{i, j}\left(\tilde{e}^{(t)}\right) \tilde{e}^{(t+1)}-u_{i, j}\left(\tilde{e}^{(t+1)}\right) \tilde{e}^{(t)}}{\tilde{e}^{(t+1)}-\tilde{e}^{(t)}} .
$$

We call this kind of approximation point-to-point approximation as the piece-wise linear approximation agrees with the original function at the end points of each interval. The following lemma shows that the above algorithm does indeed give a close approximation.

Lemma 11. For every bidder-item pair $(i, j) \in I \times J$ the algorithm described above gives a pointto-point approximation using $O\left(\left|\bar{p}_{i, j}-r_{j}\right| \cdot \sqrt{B} \cdot 1 / \sqrt{\epsilon}\right)=O(\sqrt{1 / \epsilon})$ segments that (i) is piece-wise linear, (ii) continuous, and (iii) has error at most $\epsilon$.

Proof. Point (i) is trivial as $\tilde{u}_{i, j}\left(p_{j}\right)$ is linear on each segment. Point (ii) holds as $\tilde{u}_{i, j}\left(\tilde{e}_{i, j}^{(t)}\right)=u_{i, j}\left(\tilde{e}_{i, j}^{(t)}\right)$ and $\tilde{u}_{i, j}\left(\tilde{e}_{i, j}^{(t+1)}\right)=u_{i, j}\left(\tilde{e}_{i, j}^{(t+1)}\right)$ so that for two adjacent segments the approximation agrees on the boundaries. For Point (iii) we will argue as follows.

Given that the line segment $\tilde{u}_{i, j}\left(p_{j}\right)=v_{i, j}-c_{i, j} \cdot p_{j}$ intersects the function $u_{i, j}\left(p_{j}\right)$ at points $\tilde{e}_{i, j}^{(t)}$ and $\tilde{e}_{i, j}^{(t+1)}>\tilde{e}_{i, j}^{(t)}$, what is the maximum $\max _{p_{j} \in\left[\tilde{e}_{i, j}^{(t)} \tilde{e}_{i, j}^{(t+1)}\right]}\left|u_{i, j}\left(p_{j}\right)-\tilde{u}_{i, j}\left(p_{j}\right)\right|$ for any continuous function $u_{i, j}\left(p_{j}\right)$ with a bounded second derivative? Let us define $g_{i, j}\left(p_{j}\right)=u_{i, j}\left(p_{j}\right)-\tilde{u}_{i, j}\left(p_{j}\right)$. Note that $g_{i, j}\left(p_{j}\right)$ is continuous, satisfies $g_{i, j}\left(\tilde{e}_{i, j}^{(t)}\right)=g_{i, j}\left(\tilde{e}_{i, j}^{(t+1)}\right)=0$, and is twice differentiable. Denote the first and second derivative of $g_{i, j}(\cdot)$ by $\dot{g}_{i, j}(\cdot)$ and $\ddot{g}_{i, j}(\cdot)$. Suppose $g_{i, j}\left(p_{j}\right)$ reaches an extreme point at $p_{j}=\tilde{e}_{i, j}^{(t+1)}-\delta \in\left[\tilde{e}_{i, j}^{(t)}, \tilde{e}_{i, j}^{(t+1)}\right]$ with $\dot{g}_{i, j}\left(\tilde{e}_{i, j}^{(t+1)}-\delta\right)=0$. Then, for $\tilde{e}_{i, j}^{(t+1)}-\delta \leq p_{j} \leq \tilde{e}_{i, j}^{(t+1)}$, 
$\left|\dot{g}_{i, j}\left(p_{j}\right)\right| \leq\left|\dot{g}_{i, j}\left(\tilde{e}_{i, j}^{(t+1)}-\delta\right)\right|+\left|\int_{\tilde{e}_{i, j}^{(t+1)}-\delta}^{p_{j}} \ddot{g}_{i, j}(z) d z\right| \leq \int_{\tilde{e}_{i, j}^{(t+1)}-\delta}^{p_{j}}\left|\ddot{g}_{i, j}(z)\right| d z \leq\left(p_{j}+\delta-\tilde{e}_{i, j}^{(t+1)}\right) \cdot B$. And so the maximum of $\left|g_{i, j}\left(p_{j}\right)\right|$ between $\tilde{e}_{i, j}^{(t+1)}-\delta$ and $\tilde{e}_{i, j}^{(t+1)}$ is

$$
\begin{aligned}
g_{i, j}\left(\tilde{e}_{i, j}^{(t+1)}\right)+\int_{\tilde{e}_{i, j}^{(t+1)}}^{p_{j}=\tilde{e}_{i, j}^{(t+1)}-\delta} \dot{g}_{i, j}\left(p_{j}\right) d p_{j} & \leq\left|\int_{p_{j}=\tilde{e}_{i, j}^{(t+1)}-\delta}^{\tilde{e}_{i, j}^{(t+1)}}\right| \dot{g}_{i, j}\left(p_{j}\right)\left|d p_{j}\right| \\
& \leq\left|\int_{p_{j}=\tilde{e}_{i, j}^{(t+1)}-\delta}^{\tilde{e}_{i, j}^{(t+1)}}\left(p_{j}+\delta-\tilde{e}_{i, j}^{(t+1)}\right) \cdot B d p_{j}\right| \\
& =\left(\left(\left(\tilde{e}_{i, j}^{(t+1)}\right)^{2}-\left(\delta-\tilde{e}_{i, j}^{(t+1)}\right)^{2}\right) / 2+\delta\left(\delta-\tilde{e}_{i, j}^{(t+1)}\right)\right) \cdot B \\
& =\delta^{2} / 2 \cdot B .
\end{aligned}
$$

Similarly, the maximum of $\left|\dot{g}_{i, j}\left(p_{j}\right)\right|$ in the interval $\left[\tilde{e}_{i, j}^{(t)}, \tilde{e}_{i, j}^{(t+1)}-\delta\right]$ is $\left(\tilde{e}_{i, j}^{(t+1)}-\tilde{e}_{i, j}^{(t)}-\delta\right)^{2} B / 2$. As $g_{i, j}\left(p_{j}\right)$ is continuous, the maximum achieved at the point $\tilde{e}_{i, j}^{(t+1)}-\delta$ coming from the left, increasing from $g_{i, j}\left(\tilde{e}_{i, j}^{(t)}\right)=0$, has to equal the maximum achieved at this point coming from the right, increasing from $g_{i, j}\left(\tilde{e}_{i, j}^{(t+1)}\right)=0$. So $\left|g_{i, j}\left(p_{j}\right)\right|$ is bounded by $\min \left(\left(\tilde{e}_{i, j}^{(t+1)}-\tilde{e}_{i, j}^{(t)}-\delta\right)^{2} B / 2, \delta^{2} B / 2\right)$, which is maximized for $\delta=\left(\tilde{e}_{i, j}^{(t+1)}-\tilde{e}_{i, j}^{(t)}\right) / 2$, corresponding to a peak of at most $\left(\tilde{e}_{i, j}^{(t+1)}-\tilde{e}_{i, j}^{(t)}\right)^{2} / 8 \cdot B \leq$ $w^{2} / 8 \cdot B \leq \epsilon$ by the choice of $w$.

Approximately bidder optimal outcome Given a set of continuous, piece-wise linear, pointto-point approximations $\tilde{u}_{i, j}(\cdot)$ with error at most $\epsilon$, we can use the mechanism from Section 3 to obtain a bidder optimal outcome $(\tilde{\mu}, \tilde{p})$ for the approximated input. The following lemma bounds how "far" away from the bidder optimal outcome $(\mu, p)$ for the original utility functions $u_{i, j}(\cdot)$ this outcome can be depending on how "close" the piece-wise linear approximations are, i.e., depending on how small $\epsilon$ is. The idea is as follows: Suppose we knew the bidder optimal prices $p$ for $u_{i, j}(\cdot)$. We could use them to "jumpstart" the mechanism from Section 3 . That is, starting from $p$ we could find the smallest prices $\tilde{p}^{\prime}$ such that $\left(\tilde{\mu}^{\prime}, \tilde{p}^{\prime}\right)$ is envy free for $\tilde{u}_{i, j}(\cdot)$. From Lemma 1 we know that for the bidder optimal prices $\tilde{p}$ we have that $\tilde{p} \leq \tilde{p}^{\prime}$ so that any upper bound on $\tilde{p}^{\prime}$ also applies to $\tilde{p}$. To bound the difference between $p$ and $\tilde{p}^{\prime}$ we first bound the ratio between the biggest and the smallest non-zero entry of an overdemand preserving price increase by $O\left((M / m)^{\min (n, k)}\right)$. We then argue that between any two consecutive executions of Step 7(a) in the mechanism from Section 3 this difference is increased by a multiplicative factor of $O\left((M / m)^{\min (n, k)}\right)$. The crucial point here is that the number of executions of Step 7(a) is $O\left(\min (n, k)^{2}\right)$ and thus independent of $\epsilon$, which leads to a difference between $p$ and $\tilde{p}^{\prime}$ linear in $\epsilon$. We use the resulting bound on the difference between the prices to bound the difference between the utilities. Theorem 3 follows from this bound for $\epsilon$ small enough.

Lemma 12. For every envy free outcome $(\mu, p)$ for $u_{i, j}(\cdot)$ and continuous, piece-wise linear, pointto-point approximation $\tilde{u}_{i, j}(\cdot)$ with error at most $\epsilon$ the mechanism from Section 3 finds an envy free outcome $(\tilde{\mu}, \tilde{p})$ for $\tilde{u}_{i, j}(\cdot)$ with $\tilde{p}_{j} \leq p_{j}+(2 M / m)^{(\min (n, k)+1)^{3}} \cdot \epsilon / M$ for all $j$ and $\tilde{u}_{i, \tilde{\mu}(i)}\left(\tilde{p}_{\tilde{\mu}(i)}\right)+$ $(2 M / m)^{(\min (n, k)+1)^{3}} \cdot \epsilon \geq u_{i, \mu(i)}\left(p_{\mu(i)}\right)$ for all $i$.

Proof. Consider an arbitrary envy free outcome $(\mu, p)$ for $u_{i, j}(\cdot)$. Denote the set of bidders that are unmatched under $\mu$ by $U$. Let $p_{j}^{\prime}=\max \left(p_{j}, \min \left\{\hat{p}_{j}: \forall i \in U: \tilde{u}_{i, j}\left(\hat{p}_{j}\right) \leq o_{i}\right\}\right)$. If $u_{i, j}\left(p_{j}\right) \leq o_{i}$ then $\tilde{u}_{i, j}\left(p_{j}\right) \leq o_{i}+\epsilon$ and so $\tilde{u}_{i, j}\left(p_{j}+\epsilon / m\right) \leq o_{i}$. In other words, $p_{j}^{\prime} \leq p_{j}+\epsilon / m$ for all $j$. Moreover, 
for every envy free outcome for $\tilde{u}_{i, j}(\cdot)$ with prices higher or equal to $p_{j}^{\prime}$ for all $j$ we can match the bidders in $U$ to the dummy item.

Suppose that we use the mechanism from Section 3 to find a bidder optimal outcome for the bidders in $I \backslash U$, utility functions $\tilde{u}_{i, j}(\cdot)$, and reserve prices $r_{j}=p_{j}^{\prime}$ for all $j$. Let us write $q$ for the number of Steps 7(a) that the mechanism has encountered since the beginning of its execution. We write $p^{(q)}$ for the prices after $q$ such cases and so $p_{j}^{(0)}=p_{j}^{\prime}$. Furthermore, we write $\delta^{(q)}=\max _{j}\left(p_{j}^{(q)}-p_{j}^{(0)}\right)$. Whenever we compute the sets $S$ and $T$ we have $|T|>|S|$ by the definition of strict overdemand. Hence there exists a bidder $\hat{i} \in T$ such that $\mu(\hat{i}) \notin S$. Regardless of whether $\hat{i}$ turns out to be the bidder $i$ of the $(i, j)$ pair in the next Step 7(a) or not, we will show that the required price updates for items in $T$ to add the edge $(\hat{i}, \mu(\hat{i}))$ to the first choice graph are small. This will give us an upper bound on how much the price of any item in $S$ has to be increased until we reach Step 7(a). Let $\hat{j} \in S$ be any of $\hat{i}$ 's current first choice items before the price update, i.e., $\hat{j} \in F_{p^{(q)}}(\hat{i})$. Let us define the smallest utility gap as $\alpha^{(q)}=\min _{\hat{i} \in T: \mu(\hat{i}) \notin S} \alpha_{\hat{i}}^{(q)}$, where $\alpha_{\hat{i}}^{(q)}=\max \left(0, \tilde{u}_{\hat{i}, \hat{j}}\left(p_{\hat{j}}^{(q)}\right)-\tilde{u}_{\hat{i}, \mu(\hat{i})}\left(p_{\mu(\hat{i})}^{(q)}\right)\right)$ with $\hat{i} \in T$ such that $\mu(\hat{i}) \notin S$.

Lemma 15 in the appendix shows that the sets $S$ and $T$ do not change between consecutive Steps 7(b) and so all of $\hat{i}$ 's first choice items, which can change with each new Step 7(b), remain in the set $S$ until the next Step 7(a). This means that if all items in $S$ have their price increased by at least $\alpha^{(q)} / m$ then item $\mu(\hat{i}) \notin S$ will become a new first choice item for $\hat{i}$ and so Step 7(a) is reached. Lemma 14 in the appendix shows that to ensure a price increase of at least $\alpha^{(q)} / m$ for all items in $S$, it is sufficient if any item in $S$ has a price increase of $\alpha^{(q)}(M / m)^{\min (n, k)} \cdot 1 / m$. So we know $\delta^{(q+1)} \leq \delta^{(q)}+\alpha^{(q)}(M / m)^{k} \cdot 1 / m$. We bound $\alpha^{(q)}$ as follows:

$$
\begin{aligned}
\alpha^{(q)} \leq \alpha_{\hat{i}}^{(q)} \leq & \max _{\hat{j}}\left(\max \left(0, \tilde{u}_{\hat{i}, \hat{j}}\left(p_{\hat{j}}^{(q)}\right)-\tilde{u}_{\hat{i}, \mu(\hat{i})}\left(p_{\mu(\hat{i})}^{(q)}\right)\right)\right) \\
& \leq \max _{\hat{j}}\left(\max \left(0, \tilde{u}_{\hat{i}, \hat{j}}\left(p_{\hat{j}}^{\prime}\right)-\tilde{u}_{\hat{i}, \mu(\hat{i})}\left(p^{\prime}{ }_{\mu(\hat{i})}+\delta^{(q)}\right)\right)\right) \\
& \leq \max _{\hat{j}}\left(\max \left(0, \tilde{u}_{\hat{i}, \hat{j}}\left(p_{\hat{j}}^{\prime}\right)-\tilde{u}_{\hat{i}, \mu(\hat{i})}\left(p^{\prime}{ }_{\mu(\hat{i})}\right)\right)\right)+\delta^{(q)} M \\
& =\max _{\hat{j}}\left(\operatorname { m a x } \left(0,\left(u_{\hat{i}, \mu(\hat{i})}\left(p^{\prime}{ }_{\mu(\hat{i})}\right)-\tilde{u}_{\hat{i}, \mu(\hat{i})}\left(p^{\prime}{ }_{\mu(\hat{i})}\right)\right)-\left(u_{\hat{i}, \hat{j}}\left(p_{\hat{j}}^{\prime}\right)-\tilde{u}_{\hat{i}, \hat{j}}\left(p_{\hat{j}}^{\prime}\right)\right)\right.\right. \\
& \left.\left.\quad+\left(u_{\hat{i}, \hat{j}}\left(p_{\hat{j}}^{\prime}\right)-u_{\hat{i}, \mu(\hat{i})}\left(p^{\prime}{ }_{\mu(\hat{i})}\right)\right)\right)\right)+\delta^{(q)} M \\
& \leq 2 \epsilon+\delta^{(q)} M .
\end{aligned}
$$

Combining this with $\delta^{(q+1)} \leq \delta^{(q)}+\alpha^{(q)}(M / m)^{\min (n, k)} \cdot 1 / m$ gives $\delta^{(q+1)} \leq X \cdot \delta^{(q)}+Y$ where $X=1+(M / m)^{\min (n, k)+1}$ and $Y=2 \epsilon / m \cdot(M / m)^{\min (n, k)}$. Unrolling this gives $\delta^{(q+1)} \leq X^{2} \delta^{(q-1)}+$ $X Y+Y \leq \ldots \leq X^{q+1} \delta^{(0)}+Y \cdot\left(X^{q+1}-1\right) /(X-1)=Y \cdot\left(X^{q+1}-1\right) /(X-1) \leq 2 \epsilon / m \cdot(M / m)^{\min (n, k)}$. $\left(X^{q+1}\right) /(X-1) \leq 2 \epsilon / M \cdot\left(1+(M / m)^{\min (n, k)+1}\right)^{q+1}$. Hence the maximum price increase after $q$ occurrences of Step $7(\mathrm{a})$ is bounded by $\delta^{(q)} \leq\left(1+(M / m)^{\min (n, k)+1}\right)^{q} \cdot 2 \epsilon / M$.

We claim that $q$ is at most $\min (n, k)^{2}$ and we argue as follows. In the continuous case bidders never get unmatched and the outer while-loop matches a new bidder each time it gets executed. Since there are at $\operatorname{most} \min (n, k)$ bidders this shows that the outer while-loop gets executed at $\operatorname{most} \min (n, k)$ times. Between two consecutive executions of the outer while-loop there can be at $\operatorname{most} \min (n, k)$ executions of the inner while-loop corresponding to Step 7 (a) because each such step adds an edge from a bidder $i \in T$ to an item $j \notin S$. As a consequence either bidder $i_{0}$ gets matched or the maximal alternating tree grows by at least one bidder-item pair. The former can happen exactly once. The latter can happen at $\operatorname{most} \min (n, k)$ times because there are at most 
$\min (n, k)$ bidder-item pairs.

Given that the prices for the approximated input $\tilde{u}_{i, j}(\cdot)$ cannot be much higher than for the input $u_{i, j}(\cdot)$, we can bound the utility difference as follows:

$$
\begin{aligned}
\tilde{u}_{i, \tilde{\mu}(i)}\left(\tilde{p}_{\tilde{\mu}(i)}\right) & \geq \tilde{u}_{i, \mu(i)}\left(\tilde{p}_{\mu(i)}\right) \\
& \geq \tilde{u}_{i, \mu(i)}\left(p^{\prime}{ }_{\mu(i)}+\delta^{(q)}\right) \\
& \geq \tilde{u}_{i, \mu(i)}\left(p_{\mu(i)}+\epsilon / m+2 \epsilon / M \cdot\left(1+(M / m)^{\min (n, k)+1}\right)^{q}\right) \\
& \geq \tilde{u}_{i, \mu(i)}\left(p_{\mu(i)}\right)-\epsilon M / m-\epsilon(2 M / m)^{(\min (n, k)+1)\left(\min (n, k)^{2}\right)} \\
& \geq u_{i, \mu(i)}\left(p_{\mu(i)}\right)-\epsilon-\epsilon M / m-\epsilon(2 M / m)^{(\min (n, k)+1)\left(\min (n, k)^{2}\right)} \\
& \geq u_{i, \mu(i)}\left(p_{\mu(i)}\right)-\epsilon(2 M / m)-\epsilon(2 M / m)^{(\min (n, k)+1)\left(\min (n, k)^{2}\right)} \\
& \geq u_{i, \mu(i)}\left(p_{\mu(i)}\right)-\epsilon 2 \cdot(2 M / m)^{(\min (n, k)+1)\left(\min (n, k)^{2}\right)} \\
& \geq u_{i, \mu(i)}\left(p_{\mu(i)}\right)-\epsilon \cdot(2 M / m)^{1+(\min (n, k)+1)\left(\min (n, k)^{2}\right)} \\
& \geq u_{i, \mu(i)}\left(p_{\mu(i)}\right)-(2 M / m)^{(\min (n, k)+1)^{3}} \cdot \epsilon .
\end{aligned}
$$

Here we used the bound $2\left(1+(M / m)^{\min (n, k)+1}\right) \leq 4(M / m)^{\min (n, k)+1} \leq(2 M / m)^{\min (n, k)+1}$ for $\min (n, k) \geq 1$ and that $M / m \geq 1$. Exactly the same type of manipulations can be applied to show that $\left.\tilde{p}_{j} \leq p_{j}^{\prime}+\delta^{(q)} \leq p_{j}+\epsilon / m+2 \epsilon / M \cdot\left(1+(M / m)^{\min (n, k)+1}\right)^{q}\right) \leq p_{j}+(2 M / m)^{(\min (n, k)+1)^{3}} \cdot \epsilon / M$.

Theorem 3. Given strictly monotonically decreasing, continuous utility functions $u_{i, j}(\cdot)$ satisfying assumptions (A.1) to (A.3), we can compute a $\gamma$-bidder optimal outcome with the running time specified in Theorem 1, where $D=0, T=O(\sqrt{1 / \epsilon})$, and $\epsilon=\gamma \cdot(2 M / m)^{-(\min (n, k)+1)^{3}}$.

Proof. Let $\epsilon=\gamma(2 M / m)^{-(\min (n, k)+1)^{3}}$. For each utility function $u_{i, j}(\cdot)$, find a piece-wise linear approximation $\tilde{u}_{i, j}(\cdot)$ with error at most $\epsilon$. This generates $O\left(n k \cdot|\bar{p}-r| \sqrt{B}(2 M / m)^{(\min (n, k)+1)^{3} / 2} / \sqrt{\gamma}\right)$ linear segments by Lemma 11, where $|\bar{p}-r|=\max _{i, j}\left|\bar{p}_{i, j}-r_{j}\right|$. For this approximated input we then obtain a bidder optimal outcome $(\tilde{\mu}, \tilde{p})$ in time linear in the total number of segments times $\min (n, k)^{3}$ by Theorem 1, i.e., in time $O\left(n k \cdot|\bar{p}-r| \sqrt{B}(2 M / m)^{(\min (n, k)+1)^{3} / 2} \min (n, k)^{3} / \sqrt{\gamma}\right)$. We claim that this outcome is $\gamma$-bidder optimal for $u_{i, j}(\cdot)$.

We have $\tilde{p}_{j} \geq r_{j}$ and $\tilde{p}_{j_{0}}=0$ as (1) we did not change the reserve prices and (2) we did not approximate $u_{i, j_{0}}\left(p_{j_{0}}\right)$. And so $(\tilde{\mu}, \tilde{p})$ is feasible for $u_{i, j}(\cdot)$. It is also $\gamma$-envy free as $\epsilon \leq \gamma / 2$, and so we know that $\max _{i}\left(\max _{j}\left(u_{i, j}\left(\tilde{p}_{j}\right)-u_{i, \tilde{\mu}(i)}\left(\tilde{p}_{\tilde{\mu}(i)}\right)\right)\right) \leq \max _{i}\left(\max _{j}\left(\tilde{u}_{i, j}\left(\tilde{p}_{j}\right)-\tilde{u}_{i, \tilde{\mu}(i)}\left(\tilde{p}_{\tilde{\mu}(i)}\right)+2 \epsilon\right)\right) \leq \gamma$ by the choice of $\epsilon$. Finally, and most importantly, Lemma 12 shows that the utility losses with respect to the unknown bidder optimal outcome $(\mu, p)$ are bounded by $\gamma$.

\section{Externalities}

In this section we show that two natural generalizations of our problem are $\mathcal{N} \mathcal{P}$-hard: In the first, the utility functions are allowed to depend on the matching. In the second, they are allowed to depend on the vector of prices.

Utilities that depend on the matching Our first hardness result is for utility functions that depend on the matching. These utility functions allow, for example, to express that a given advertiser (e.g., Coca-Cola) achieves a higher utility if he gets an ad slot that is above the slot of her competitor (e.g., Pepsi). For utility functions of this kind it is $\mathcal{N} \mathcal{P}$-complete to decide whether there exists an outcome in which the sum of the utilities is above a certain threshold. 
Theorem 4. Given utility functions $u_{i, j}(\mu)$ that depend on the matching $\mu$, and given a constant $K$, deciding whether there exists an outcome with sum of the utilities at least $K$ is $\mathcal{N} \mathcal{P}$-complete. This is true even if there is an ordering on the items, each bidder $i$ has a set $S_{i}$ of three other bidders, and the utility of bidder $i$ depends on whether in the ordering the items to which the bidders in $S_{i}$ are matched to appear before or after the item that bidder $i$ is matched to.

Proof. Checking whether a given outcome $(\mu, p)$ has sum of the utilities at least $K$ takes polynomial time. This shows that the problem belongs to $\mathcal{N} \mathcal{P}$. We prove $\mathcal{N} \mathcal{P}$-completeness by providing a polynomial-time reduction from 3-SAT [16]: Given $n$ Boolean variables $x_{k}, m$ clauses $c_{r}$ (disjunction of up to three literals, i.e., $x_{k}$ or $\neg x_{k}$ ), and a formula $f$ (conjunction of the $m$ clauses) decide whether there exists a satisfying assignment $x$.

For a given instance of 3-SAT: For every variable create one bidder, called variable bidder, and two items, one called true item and one called false item. The variable bidder has a utility of zero for each of these items and minus infinity for all other items. For every clause create one bidder, called clause bidder, and one item, called clause item. In the ordering all true items are before all clause items, and all clause items are before all false items. The utility of a clause bidder for the clause item depends on whether the variable bidders correspoding to variables in that clause are matched to items before or after the clause item, i.e., to a true item or a false item. It is 1 if and only if the matching is such that the clause is satisfied and 0 otherwise. The utility function of a clause bidder for all other items is minus infinity.

It is not difficult to see that there is an outcome with sum of the utilities at least $K=m$ if and only if the formula is satisfiable. This completes the proof.

Utilities that depend on the vector of prices Our second hardness result concerns situations in which the utility that a bidder derives from being matched to an item depends on the whole vector of prices. These utility functions allow, for example, to express that a bidder $i$ achieves a higher utility when the other bidders have to pay more, i.e., when items that $i$ does not get become more expensive. For these utility functions deciding whether there exists an envy free outcome in which the sum of the utilities is above a certain threshold is $\mathcal{N} \mathcal{P}$-complete.

Theorem 5. Given utility functions $u_{i, j}(p)$ that depend on the whole vector of prices $p=\left(p_{1}, \ldots, p_{k}\right)$, that are monotonically decreasing in $p_{j}$ and monotonically increasing in $p_{l}$ for all $l \neq j$, and given a constant $L$, it is $\mathcal{N} \mathcal{P}$-complete to decide whether there exists an envy free outcome $(\mu, p)$ in which the sum of the utilities is at least $L$.

Proof. We prove the claim for utility functions $u_{i, j}\left(p_{1}, \ldots, p_{k}\right)$ that depend on the vector of prices $p=\left(p_{1}, \ldots, p_{k}\right)$ and are decreasing in $p_{l}$ for all $l$. The proof for utility functions $u_{i, j}\left(p_{1}, \ldots, p_{k}\right)$ that are decreasing in $p_{j}$ and increasing in $p_{l}$ with $l \neq j$ is similar.

Checking whether a given outcome $(\mu, p)$ is envy free and has sum of the utilities at least $L$ takes polynomial time. This establishes membership in $\mathcal{N} \mathcal{P}$. To prove hardness we provide a polynomialtime reduction from MAX-2-NASH [31, 15]: There are two players $i \in 1,2$. For $i=1$ we use $-i$ to denote player 2, and vice versa. Each player $i$ has a finite set of strategies $S_{i}$ and a payoff function $a_{i}(s, t)$ describing her payoff if he plays strategy $s \in S_{i}$ and the other player plays strategy $t \in S_{-i}$. A mixed strategy for player $i$ is a probability distribution $\sigma_{i}$ over $S_{i}$. A strategy profile $\sigma=\left(\sigma_{1}, \sigma_{2}\right)$ defines a mixed strategy for each of the two players. A strategy $s \in S_{i}$ is in the support of player $i$ if and only if $\sigma_{i}(s)>0$, i.e., player $i$ plays strategy $s$ with non-zero probability. A strategy profile $\sigma^{*}=\left(\sigma_{1}^{*}, \sigma_{2}^{*}\right)$ is a mixed Nash equilibrium (mixed NE) if for every player $i$ and all mixed strategies $\sigma_{i}: \sum_{s \in S_{i}}\left(\sum_{t \in S_{-i}} a_{i}(s, t) \cdot \sigma_{-i}^{*}(t)\right) \cdot \sigma_{i}^{*}(s) \geq \sum_{s \in S_{i}}\left(\sum_{t \in S_{-i}} a_{i}(s, t) \cdot \sigma_{-i}^{*}(t)\right) \cdot \sigma_{i}(s)$. A pure strategy $s$ is a best response if for all pure strategies $r: \sum_{t \in S_{-i}} a_{i}(s, t) \cdot \sigma_{-i}^{*}(t) \geq \sum_{t \in S_{-i}} a_{i}(r, t) \cdot \sigma_{-i}^{*}(t)$. It 
can be shown that $\sigma^{*}=\left(\sigma_{1}^{*}, \sigma_{2}^{*}\right)$ is a mixed NE if and only if every pure strategy in the support of $\sigma^{*}$ is a best response. The goal is to compute a mixed $\mathrm{NE} \sigma^{*}=\left(\sigma_{1}^{*}, \sigma_{2}^{*}\right)$ with maximum total payoff. The decision version of this problem asks for a mixed NE with total payoff at least $K$.

MAX-2-NASH is $\mathcal{N} \mathcal{P}$-complete even if both players have the same number of strategies $\left|S_{1}\right|=$ $\left|S_{2}\right|=N$ and all entries in the payoff matrices are integers between 0 and $N$ [31, 15]. Let $C=$ $\left(N ! \cdot N^{N}\right)^{2 N}$. Note that $C$ can be represented with at most $4 N^{2} \log (N)$ bits. Given an MAX-2NASH instance with $\left|S_{1}\right|=\left|S_{2}\right|=N$ and payoff matrices with integer entries between 0 and $N$ we construct an instance of our problem by means of the following "gadgets":

Best response gadget B: For each player $i$ and strategy $s \in S_{i}$ we add one best response gadget $B$ consisting of two bidders $i s 1$ and $i s 2$ and two items $i s 3$ and $i s 4$. Bidder $i s 1$ has a utility of $u_{i s 1, i s 3}(p)=\sum_{t \in S_{-i}} a_{i}(s, t) \cdot\left(1-p_{-i t 3}\right)$ for item $i s 3$ and a utility of $u_{i s 1, i s 4}(p)=\max _{t \in S_{i}} u_{i t 1, i t 3}(p)$ for item $i s 4$. Bidder $i s 2$ has a utility of $u_{i s 2, i s 4}(p)=1 /(8 N C)-p_{i s 4}$ for item $i s 4$. Both bidders have an outside option of 0 , i.e., $o_{i s 1}=o_{i s 2}=0$.

Probability gadget P1: For each player $i$ and strategy $s \in S_{i}$ we add one probability gadget $P 1$ consisting of two bidders isa and isb and two items isc and isd. Bidder isa has a utility of 0 for item $i s c$, and a utility of $1-p_{i s 3}$ for $i s d$. Bidder $i s b$ has a utility of 1 for item $i s c$, and a utility of $-\infty$ for $i s d$. The outside options are $-\infty$.

Probability gadget P2: For each player $i$ and strategy $s \in S_{i}$ we add one probability gadget P2 consisting of two bidders $i s a^{\prime}$ and $i s b^{\prime}$ and two items $i s c^{\prime}$ and $i s d^{\prime}$. Bidder $i s a^{\prime}$ has a utility of 0 for item $i s c^{\prime}$, and a utility of $1 /(8 N C)-p_{i s 4}$ for $i s d^{\prime}$. Bidder $i s b^{\prime}$ has a utility of 1 for item $i s c^{\prime}$, and a utility of $-\infty$ for $i s d^{\prime}$. The outside options are $-\infty$.

Distribution gadget D1: For each player $i$ we add one distribution gadget D1 consisting of two bidders and one item. Both bidders have a utility of $\left[\sum_{s \in S_{i}}\left(1-p_{i s 3}\right) \cdot\left(1 /(8 N C)-p_{i s 4}\right)\right]-1 /(8 N C)$ for the item and an outside option of 0 .

Distribution gadget D2: For each player $i$ we add one distribution gadget D2 consisting of two bidders and one item. Both bidders have a utility of $\left[\sum_{s \in S_{i}}\left(1-p_{i s 3}\right)\right]-1$ for the item and an outside option of 0 .

Claim 1. If all payoffs $a_{i}\left(s_{i}, s_{-i}\right)$ in a 2-player game are non-negative integers, with the largest being $N$, then the probabilities in any mixed $N E$ are rational numbers with the smallest being $1 /\left(N ! \cdot N^{N}\right)$.

Proof. Given the support of a mixed NE, the corresponding mixed NE $\sigma^{*}=\left(\sigma_{1}^{*}, \sigma_{2}^{*}\right)$ can be determined by solving a linear equation system (LES). Since the coefficients in this LES are nonnegative integers, with the largest being $N$, any solution $\sigma^{*}=\left(\sigma_{1}^{*}, \sigma_{2}^{*}\right)$ to this LES consists of rational numbers with denominator at most $N ! \cdot N^{N}$ [39]. Hence the smallest probability in the mixed NE is at least $1 /\left(N ! \cdot N^{N}\right)$.

Claim 2. There exists a mixed $N E \sigma^{*}=\left(\sigma_{1}^{*}, \sigma_{2}^{*}\right)$ with total payoff $K$ if and only if there exists an envy free outcome $(\mu, p)$ with sum of the utilities strictly larger than $N \cdot(K+4)+2$ and less than $N \cdot(K+4)+2+1 / C$.

Proof. For the only if-part assume that such a mixed NE $\sigma^{*}=\left(\sigma_{1}^{*}, \sigma_{2}^{*}\right)$ exists. Denote the support of player $i$ by $S U P P_{i}$. For each player $i$ and strategy $s$ : If $s$ is in the support of player $i$, then match $i s 1$ to $i s 3$ and $i s 2$ to $i s 4$ and set $p_{i s 3}=1-\sigma_{i}^{*}(s)<1$ and $p_{i s 4}=0$. If it is not, then match $i s 1$ to $i s 4$ and leave $i s 2$ unmatched and set $p_{s 3}=1-\sigma_{i}^{*}(s)=1$ and $p_{i s 4}=1 /(8 N C)$. In the former case this is envy free because $s$ is one of $i$ 's best responses and so $u_{i s 1, i s 3}(p) \geq u_{i s 1, i s 4}(p)$ and at $p_{i s 4}=0$ 
we have $u_{i s 2, i s 4}(p) \geq 0$. In the latter case this is envy free because $u_{i s 1, i s 4}(p) \geq u_{i s 1, i s 3}(p)$ and at $p_{i s 4}=1 /(8 N C)$ we have $u_{i s 2, i s 4}(p)=0$. Since $0 \leq p_{i s 3} \leq 1$ for all $i s 3$ items and $0 \leq p_{i s 4} \leq 1 /(8 N C)$ for all $i s 4$ items, we can match the bidders in the $P 1$ and $P 2$ gadgets in an envy free manner. Since (a) $\sum_{s \in S_{i}}\left(1-p_{i s 3}\right)=1$ and (b) $1-p_{i s 3}=0$ if and only if $1 /(8 N C)-p_{i s 4}=0$, this is also true for the bidders in the $D 1$ and $D 2$ gadgets. The $i s 1$ vertices contribute a utility of $N \cdot K$, namely $K_{1}$ each for the ones of player 1 and $K_{2}$ each for the ones of player 2 with $K_{1}+K_{2}=K$. The $i s 2$ vertices contribute $\left(\left|S U P P_{1}\right|+\left|S U P P_{2}\right|\right) /(8 N C)$. The $P 1$ gadgets contribute $2 \cdot N+2$. The $P_{2}$ gadgets contribute $2 \cdot N+\left(\left|S U P P_{1}\right|+\left|S U P P_{2}\right|\right) /(8 N C)$. The distribution gadgets contribute nothing. Hence the total utility is $N \cdot(K+4)+2+\left(\left|S U P P_{1}\right|+\left|S U P P_{2}\right|\right) /(4 N C)$. The claim follows from the fact that $1 \leq\left|S U P P_{i}\right| \leq N$ for both $i$ and, thus, $2 \leq\left|S U P P_{1}\right|+\left|S U P P_{2}\right| \leq 2 N$.

For the if-part consider an envy free outcome that maximizes the sum of the utilities. Denote the sum of the utilities by $L$. Let $T_{1}=\left\{s \in S_{1}: i s 1\right.$ is matched to $\left.i s 3\right\}$ and let $T_{2}=\left\{s \in S_{2}\right.$ : $i s 1$ is matched to $i s 3\}$. Let $K_{1}$ denote the utility of each of the $1 s 1$ players and let $K_{2}$ denote the utility of each of the $2 s 1$ players. Let $K=K_{1}+K_{2}$. It follows that $L=N \cdot(K+4)+2+\left(\left|T_{1}\right|+\right.$ $\left.\left|T_{2}\right|\right) /(8 N C)$. Since $\left|T_{1}\right| \leq N$ and $\left|T_{2}\right| \leq N$, this is at most $N \cdot(K+4)+2+1 / C$. We show below that for each player $i$ with $p_{i s 3}<1$ bidder $i s 1$ must be matched to $i s 3$. Due to the distribution gadget $D 2, \sum_{s \in S_{i}}\left(1-p_{i s 3}\right)=1$. Thus there is at least one strategy $s$ such that $1-p_{i s 3}>0$, i.e., $p_{i s 3}<1$. It follows that for each player there is at least one $i s 1$ node that is matched to an $i s 3$ node. In other words, $\left|T_{1}\right|+\left|T_{2}\right| \geq 2$ and, thus, $L>N \cdot(K+4)+2$.

Next we show that $\sigma^{*}=\left(\left(1-p_{1 s 3}\right)_{s \in S_{1}},\left(1-p_{2 s 3}\right)_{s \in S_{2}}\right)$ is a mixed NE, i.e., for each player $i$ every strategy $s \in S_{i}$ with $1-p_{i s 3}>0$ is a best response to $\sigma_{-i}^{*}=\left(1-p_{-i s 3}\right)_{s \in S_{-i}}$. We do this by showing that (1) if $1-p_{i s 3}>0$, then $i s 1$ is matched to $i s 3$, and (2) if $i s 1$ is matched to $i s 3$, then $s$ is a best response to $\sigma_{-i}^{*}=\left(1-p_{-i s 3}\right)_{s \in S_{-i}}$.

To (1): To show that if $\left(1-p_{i s 3}\right)>0$, then $i s 1$ is matched to $i s 3$ we show that if $i s 1$ is not matched to $i s 3$, then $\left(1-p_{i s 3}\right) \leq 0$. Note that by envy freeness if $i s 1$ is not matched to $i s 3$, then it must be matched to $i s 4$. Also note that by $\mathrm{P} 1$ we have $\left(1-p_{i s 3}\right) \geq 0$ so that $\left(1-p_{i s 3}\right) \leq 0$ is equivalent to $p_{i s 3}=1$. In other words, we have to show that if $i s 1$ is matched to $i s 4$, then $p_{i s 3}=1$. If $i s 1$ is matched to $i s 4$, then $p_{i s 4} \geq 1 /(8 N C)$ because otherwise $i s 2$ is not envy free. With $P 2$ we know that $p_{i s 4}=1 /(8 N C)$. Additionally, since the outcome maximizes the sum of the utilities, if $i s 1$ is matched to $i s 3$, then $p_{i s 4}=0$. Now $D 1$ guarantees: $\sum_{s \in S_{i}, i s 1}$ is matched to $i s 3\left(1-p_{i s 3}\right)=1$. And $D 2$ guarantees: $\sum_{s \in S_{i}}\left(1-p_{i s 3}\right)=1$. We get $\sum_{s \in S_{i}, i s 1 \text { matched to } i s 4}\left(1-p_{i s 3}\right)=0(\star)$. Since by $P 1$ we know that $1-p_{i s 3} \geq 0$ for all $i$ and $s,(\star)$ is only possible if $\left(1-p_{i s 3}\right)=0$ for all $s \in S_{i}$ with $i s 1$ matched to $i s 4$. This shows that if $i s 1$ is matched to $i s 4$, then $p_{i s 3}=1$.

To (2): If $i s 1$ is matched to $i s 3$, then $u_{i s 1, i s 3}(p) \geq u_{i s 1, i s 4}(p)$ because otherwise the outcome would not be envy free. This shows that $\sum_{t \in S_{-i}} a_{i}(s, t) \sigma_{-i}^{*}(t) \geq \max _{r \in S_{i}} \sum_{t \in S_{-i}} a_{i}(r, t) \sigma_{-i}^{*}(t)$. Thus, for all $r \in S_{i}$ : $\sum_{t \in S_{-i}} a_{i}(s, t) \sigma_{-i}^{*}(t) \geq \sum_{t \in S_{-i}} a_{i}(r, t) \sigma_{-i}^{*}(t)$. In other words, $s$ is a best response to $\sigma_{-i}^{*}=\left(1-p_{i t 3}\right)_{t \in S_{-i}}$.

We conclude by showing that the payoff of the mixed NE $\sigma^{*}=\left(\left(1-p_{1 s 3}\right)_{s \in S_{1}},\left(1-p_{2 s 3}\right)_{s \in S_{2}}\right)$ is $K$. The payoff to player $i$ is $u_{i}\left(s, \sigma_{-i}^{*}\right)=\sum_{t \in S_{-i}} a_{s, t}(s, t) \sigma_{-i}^{*}(t)$, where $s$ is some strategy in $i$ 's support. For every such strategy $s$, we have $\sigma_{-i}^{*}(t)=\left(1-p_{-i t 3}\right)$ and, thus, $u_{i}\left(s, \sigma_{-i}^{*}\right)=$ $\sum_{t \in S_{-i}} a_{s, t}(s, t)\left(1-p_{-i t 3}\right)=K_{i}$. Since this is true for both $i$ the total payoff is $K_{1}+K_{2}=K$.

\section{Future Work}

The demand for more expressive mechanisms is reflected in the richness of preferences offered by web applications as diverse as matchmaking sites, sites like Amazon and NetFlix, and services like 
Google's AdSense. Standard mechanisms often do not meet this demand. Providing mechanisms that do meet this demand and that at the same time (1) guarantee the existence of a stable solution, (2) are computationally tractable, and (3) have good incentive properties is one of the major challenges that the field of computational mechanism design is currently facing.

In this paper we contributed to this general agenda by considering the domain of multi-item auctions with unit demand and by providing the most expressive mechanism for this setting so far. This mechanism, which can be seen as a generalization of the General Auction Mechanism of [1], can handle piece-wise linear utility functions with non-identical slopes and multiple discontinuities. These utility functions allow the bidders to explicitly specify conversion rates (enabling, e.g., per-click auctions that are simultaneously envy free for bidders with per-click and per-impression valuations) and a variety of soft and hard budget constraints (which, e.g., arise when bidders have a limited amount of cash and have to take out loans). An interesting direction for future work would be to push the "expressiveness frontier" even further. This is particularly true for more general domains, e.g., one-to-many and many-to-many domains.

On a more abstract level it would be desirable to have a "theory of expressiveness", which helps to find the right degree of expressiveness. More expressiveness might be good (e.g., because more efficient outcomes are obtainable), but it might also be too much (e.g., stable outcomes may no longer exist, may be hard to compute, or may be easy to manipulate). A first step towards such a general theory was recently undertaken by [9, 10] and [41, 23]. Any refinement of this theory would be highly interesting.

\section{References}

[1] G. Aggarwal, S. Muthukrishnan, D. Pál, and M. Pál. General auction mechanism for search advertising. In Proc. of 18th $W W W$, pages 241-250, 2009.

[2] S. Alaei, K. Jain, and A. Malekian. Competitive equilibrium in two sided matching markets with general utility functions. SIGecom Exchanges, 10(2):Article 8, 2011.

[3] A. Alkan. Existence and computation of matching equilibria. European Journal of Political Economy, 5(2-3):285-296, 1989.

[4] M. Alkan. Equilibrium in a matching market with general preferences. In M. Majumdar, editor, Equilibrium and Dynamics. Essays in Honour of David Gale, pages 1-16. The MacMillan Press Ltd, London, 1992.

[5] I. Ashlagi, M. Braverman, and A. Hassidim. Ascending unit demand auctions with budget limits. Working paper, pages 1-11, 2009.

[6] I. Ashlagi, M. Braverman, A. Hassidim, R. Lavi, and M. Tennenholtz. Position auctions with budgets: Existence and uniqueness. The B.E. Journal of Theoretical Economics, 10(1):Advances, Article 20, 2010.

[7] I. Ashlagi, P. Krysta, and M. Tennenholtz. Social context games. In Proc. of 4th WINE, pages 675-683, 2008.

[8] P. Bajari and A. Hortacsu. The winner's curse, reserve prices, and endogenous entry: Empirical insights from ebay auctions. The RAND Journal of Economics, 34(2):329-355, 2003.

[9] M. Benisch, N. Sadeh, and T. Sandholm. A theory of expressiveness in mechanisms. In Proc. of 23rd AAAI, pages 17-23, 2008. 
[10] M. Benisch, N. Sadeh, and T. Sandholm. Methodology for designing reasonably expressive mechanisms with application to ad auctions. In Proc. of 21st IJCAI, pages 46-52, 2009.

[11] S. Borgs, J. Chayes, N. Immorlica, M. Mahdian, and A. Saberi. Multi-unit auctions with budget constrained bidders. In Proc. of 6th EC, pages 44-51, 2005.

[12] N. Chen, X. Deng, and A. Ghosh. Competitive equilibria in matching markets with budgets. SIGecom Exchanges, 9(1):Article 5, 2010.

[13] X. Chen and X. Deng. Settling the complexity of two-player nash equilibrium. In Proc. of 4 7th FOCS, pages 261-272, 2006.

[14] E. H. Clarke. Multipart pricing of public goods. Public Choice Journal, 11(1):17-33, 1971.

[15] V. Conitzer and T. Sandholm. Complexity results about nash equilibria. Proc. of 18th IJCAI, pages $765-771,2003$.

[16] S. A. Cook. The complexity of theorem-proving procedures. In Proc. of 3rd STOC, pages 151-158, 1971.

[17] C. Daskalakis and C. H. Papadimitriou. Three-player games are hard. Electronic Colloquium on Computational Complexity, pages 1-10, 2005.

[18] G. Demange and D. Gale. The strategy structure of two-sided matching markets. Econometrica, 53(4):873-888, 1985.

[19] G. Demange, D. Gale, and M. Sotomayor. Multi-item auctions. Journal of Political Economy, 94(4):863-72, 1986.

[20] G. Demange, D. Gale, and M. Sotomayor. A further note on the stable matching problem. Discrete Applied Mathematics, 16(3):217-222, 1987.

[21] X. Deng and Y. Du. The computation of approximate competitive equilibrium is PPAD-hard. Information Processing Letters, 108(6):369-373, 2008.

[22] S. Dobzinski, R. Lavi, and N. Nisan. Multi-unit auctions with budget limits. In Proc. of 49th FOCS, pages 260-269, 2008.

[23] P. Dütting, F. Fischer, and D. C. Parkes. Simplicity-expressiveness tradeoffs in mechanism design. In Proc. of 12th EC, pages 341-350, 2011.

[24] P. Dütting, M. Henzinger, and I. Weber. Bidder optimal assignments for general utilities. In Proc. of 5th WINE, pages 575-582, 2009.

[25] P. Dütting, M. Henzinger, and I. Weber. Sponsored search, market equilibria, and the hungarian method. In Proc. of 27th STACS, pages 287-298, 2010.

[26] B. Edelman, M. Ostrovsky, and M. Schwarz. Internet advertising and the generalized second price auction: Selling billions of dollars worth of keywords. American Economic Review, 97(1):242-259, 2007.

[27] J. Edmonds and R. M. Karp. Theoretical improvements in algorithmic efficiency for network flow problems. Journal of the ACM, 19(2):248-264, 1972. 
[28] J. Feldman, M. Henzinger, N. Korula, V. Mirrokni, and C. Stein. Online stochastic packing applied to display ad allocation. In Proc. of 18th ESA, pages 1-13, 2010.

[29] D. Gale and M. Sotomayor. Some remarks on the stable matching problem. Discrete Applied Mathematics, 11(3):223-232, 1985.

[30] A. Ghosh and A. Sayedi. Expressive auctions for externalities in online advertising. In Proc. of 19th $W W W$, pages 371-380, 2010.

[31] I. Gilboa and E. Zemel. Nash and correlated equilibria: Some complexity considerations. Games and Economic Behavior, 1(1):80-93, 1989.

[32] A. Goel and K. Munagala. Hybrid keyword search auctions. In Proc. of 18th WWW, pages 221-230, 2009.

[33] T. Groves. Incentives in teams. Econometrica, 41(4):617-631, 1973.

[34] P. Hall. On representatives of subsets. London Mathematical Society, 10:26-30, 1935.

[35] P. Jehiel, B. Moldovanu, and E. Stacchetti. How (not) to sell nuclear weapons. American Economic Review, 86(4):814-29, 1996.

[36] D. Kempe, A. Mu'alem, and M. Salek. Envy-free allocations for budgeted bidders. In Proc. of 5th WINE, pages 537-544, 2009.

[37] H. W. Kuhn. The Hungarian Method for the assignment problem. Naval Research Logistics, 2:83-97, 1955.

[38] H. B. Leonard. Elicitation of honest preferences for the assignment of individuals to positions. Journal of Political Economy, 91(3):461-479, 1983.

[39] C. Limongelli and R. Pirastu. Exact solution of linear systems over rational numbers by parallel p-adic arithmetic. Proc. of 3rd CONPAR, pages 313-323, 1994.

[40] A. Mehta, A. Saberi, U. Vazirani, and V. Vazirani. Adwords and generalized online matching. Journal of the ACM, 54(5):1-19, 2007.

[41] P. Milgrom. Simplified mechanisms with an application to sponsored-search auctions. Games and Economic Behavior, 70(1):62-70, 2010.

[42] N. Nisan, T. Roughgarden, É. Tardos, and V. Vazirani. Algorithmic Game Theory. Cambridge University Press, New York, 2007.

[43] A. E. Roth and A. Ockenfels. Last-minute bidding and the rules for ending second-price auctions: Evidence from ebay and amazon auctions on the internet. The American Economic Review, 92(4):1093-1103, 2002.

[44] A. Schrijver. Theory of Linear and Integer Programming. John Wiley \& Sons, New York, 1998.

[45] L. S. Shapley and M. Shubik. The assignment game: The core I. International Journal of Game Theory, 1(1):111-130, 1971.

[46] N. Tomizawa. On some techniques useful for solution of transportation network problems. Networks, 1(2):173-194, 1971. 
[47] H. Varian. Position auctions. International Journal of Industrial Organization, 25(6):11631178, 2007.

[48] V. Vazirani and M. Yannakakis. PPAD-completeness of fisher markets under piecewise-linear, concave utility functions. In Proc. of 6th ICTS, pages 156-166, 2010.

[49] W. Vickrey. Counterspeculation, auctions, and competitive sealed tenders. Finance, 16(1):8$27,1961$.

\section{A Analysis of Mechanism for Continuous Utility Functions}

The following lemmata bound the price increase between two consecutive Steps 7(a) in the mechanism for piece-wise linear utility functions when there are no discontinuities.

Lemma 13. Let $\mathcal{T}, S$, and $T$ be the maximal alternating tree, item set, and bidder set considered by the mechanism. Then the overdemand-preserving price increase d computed in l.6 satisfies $\max _{j^{\prime} \in S, j \in S}\left(d_{j^{\prime}} / d_{j}\right) \leq(M / m)^{\min (n, k)}$.

Proof. Let $j$ and $j^{\prime}$ be two distinct elements in $S$. As $\mathcal{T}$ is a tree we know that $j$ and $j^{\prime}$ are connected by a path, alternating between items in $S$ and bidders in $T$, of length at most $2 \min (|S|,|T|) \leq$ $2 \min (n, k)$. Let this path be $j=j_{0}, i_{0}, j_{1}, i_{1}, j_{2}, \ldots, j_{s-1}, i_{s-1}, j_{s}=j^{\prime}$. From Lemma 5 we know that as $d$ is envy free for $\mathcal{T}$ we must have both $c_{t, t} \cdot d_{t} \leq c_{t, t+1} \cdot d_{t+1}$ and $c_{t, t} \cdot d_{t} \geq c_{t, t+1} \cdot d_{t+1}$, and hence $c_{t, t} \cdot d_{t}=c_{t, t+1} \cdot d_{t+1}$ for all $0 \leq t<s$. Applying this to the whole path we get $d_{j^{\prime}}=d_{j} \prod_{t=0}^{s-1}\left(c_{t, t} / c_{t, t+1}\right) \leq(M / m)^{\min (n, k)} d_{j}$.

Lemma 14. Let $d^{(1)}, \ldots, d^{(s)}$ be a sequence of consecutive overdemand preserving price increases (l.6) such that the set $S$ (l.4) is the same for all of them. Let $\lambda^{(1)}, \ldots, \lambda^{(s)}$ be the corresponding scalars (l.7). Then for any $j, j^{\prime} \in S$ we have $\left(\sum_{l=1}^{s} \lambda^{(l)} d_{j}^{(l)}\right) /\left(\sum_{l=1}^{s} \lambda^{(l)} d_{j^{\prime}}^{(l)}\right) \leq(M / m)^{\min (n, k)}$.

Proof. Lemma 13 ensures that for all $l \in\{1, \ldots, s\}: d_{j}^{(l)} \leq(M / m)^{\min (n, k)} d_{j^{\prime}}^{(l)}$. Hence, $\lambda^{(l)} d_{j}^{(l)} \leq$ $\lambda^{(l)}(M / m)^{\min (n, k)} d_{j^{\prime}}^{(l)}$ and the result follows by summing over all $l$.

Lemma 15. From one Step 7(a), where a new bidder-item pair $(i, j)$ enters the first choice graph, to the next, which can be separated by many Steps $7(b)$ and changes in the price increase d, the sets $S$ and $T$ considered by the mechanism do not change.

Proof. We will prove the claim by induction over the number of Steps 7(b) separating two Steps 7 (a). Let $b$ be this number. If $b=0$ then the claim holds trivially. Denote the maximum alternating tree before the $b$-th update by $\mathcal{T}^{(b)}$. This tree is with respect to the matching $\mu^{(b)}$ and spans the items in $S$ and the bidders in $T$ and is computed in 1.3 of the mechanism in Section 3. Computing the overdemand preserving price increase $d$ in 1.6 of the mechanism results in an implicitly computed new tree $\mathcal{T}^{(b)}$, the tree corresponding to $d$, spanning the same items and bidders that corresponds to a new matching $\mu^{(b)}$. Since $d$ is envy free for $\mathcal{T}^{(b)}$ and the utility functions are continuous all edges in $\mathcal{T}^{(b)}$ (and in particular those in $\mu^{\prime(b)}$ ) belong to the first choice graph after the price update (hence no edges are removed from $\mu^{\prime(b)}$ in 1.9 of the mechanism). It follows that the max alternating tree $\mathcal{T}^{(b+1)}$ that is explicitly computed in 1.10 of the mechanism is identical to $\mathcal{T}^{b}$, which we know spans the same items and bidders as $\mathcal{T}^{(b)}$, namely $S$ and $T$. 